\title{
Synthesis, characterization and screening of antimicrobial, antituberculosis, antiviral and anticancer activity of novel 1,3-thiazolidine-4-ones derived from 1-[2-(benzoylamino)-4- (methylthio)butyryl]-4-alkyl/arylalkyl thiosemicarbazides ${ }^{\dagger}$
}

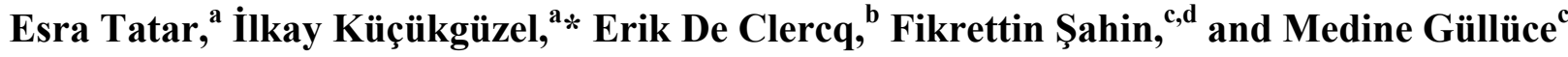 \\ ${ }^{a}$ Marmara University, Faculty of Pharmacy, Department of Pharmaceutical Chemistry, \\ Haydarpaşa 34668 Istanbul, Turkey \\ ${ }^{b}$ Rega Institute for Medical Research, Katholieke Universiteit Leuven, 10 Minderbroedersstraat, \\ 3000 Leuven, Belgium \\ ${ }^{c}$ Atatürk University, Biotechnology Application and Research Center, 25240 Erzurum, Turkey. \\ ${ }^{d}$ Yeditepe University, Faculty of Engineering and Architecture, Department of Genetic and \\ Bioengineering, 34755 Istanbul, Turkey \\ E-mail:ikucukguzel@marmara.edu.tr
}

\begin{abstract}
1,3-Thiazolidine-4-ones have been known to possess miscellaneous pharmacological activities such as antibacterial, antimycobacterial, antiviral, anticancer and anticonvulsant. Therefore, we synthesized some novel 1-[2-(benzoylamino)-4-(methylthio)butyryl]-4-alkyl/aryl alkyl thiosemicarbazides, $\quad N$-\{1-[[2-(3-Alkyl/arylalkyl-4-oxo-1,3-thiazolidin-2-ylidene) hydrazino] carbonyl-3-(methylthio)propyl $\}$ benzamides and $N-\{1-[[2-[5-$ benzylidene)-3-alkyl/arylalkyl-4oxo-1,3-thiazolidin-2-ylidene]hydrazino]carbonyl-3-(methyl-thio)propyl $\}$-benzamide derivatives. Structures of the synthesized compounds were elucidated by IR, ${ }^{1} \mathrm{H}-/{ }^{13} \mathrm{C}$ - NMR and HR-MS spectral data. All of the compounds were tested for antibacterial and antituberculosis activity and some of the compounds exhibited marginal activity against Staphylococcus aureus ATCC 29213, Bacillus subtilis A57 and Candida albicans A177. Antiviral activity of the synthesized compounds was determined against various types of viruses in HEL, HeLa and Vero cell cultures. Anti-HIV and cytotoxicity data were also obtained with the compounds using the strains HIV-1 ( $\mathrm{III}_{\mathrm{B}}$ ) and HIV-2 (ROD) in an MT-4/MTT based assay. None of the tested compounds showed antiviral activity at subtoxic doses whereas some of them exhibited remarkable cytotoxic potential. Target compounds were also screened for their antituberculosis potency against Mycobacterium tuberculosis H37 Rv, and some of them showed varying degrees of inhibition. Among the compounds tested, compound 27 was found to be most potent with $90 \%$ inhibition of mycobacterial growth at $6.25 \mu \mathrm{g} / \mathrm{ml}$. Compounds 8, 13, 14, 23, 25 were evaluated for their anticancer activity by the National Cancer Institute (NCI).
\end{abstract}

\footnotetext{
${ }^{\dagger}$ This work was partly presented at the $2^{\text {nd }}$ International Meeting on Medicinal and Pharmaceutical Chemistry, Antalya - TURKEY, P9, 10-14 October 2004.
} 
Keywords: 4-Thiazolidinones, methionine, antituberculosis activity, antiviral activity, anti-cancer activity

\section{Introduction}

The enhanced prevalence of infectious diseases threatens world population. Although tuberculosis appeared as a curable disease for years, it is regaining importance due to the multidrug resistance. ${ }^{1,2}$ Worldwide statistics on tuberculosis surprisingly reveals that, nearly one-third of the world's population is infected with tuberculosis, with approximately eight million new patients every year. ${ }^{3}$ A major issue is the increase of multi-drug resistant tuberculosis (MDRTB) giving rise to the disease expensive and incurable especially in immunodeficient subjects such as AIDS patients. ${ }^{4}$ Hence, there is an increased demand to develop new antituberculosis agents effective against pathogens resistant to current treatment.

Human immunodeficiency virus type 1 (HIV-1) has been recognized to be responsible for transmission and progress of acquired immune deficiency syndrome (AIDS). ${ }^{5}$ There are many targets for anti - HIV drug development due to the unique nature of the replication of HIV-1. Of these target macromolecules, reverse transcriptase (RT) plays an essential role in the replicative cycle of HIV-1. ${ }^{6}$ A key step for the replication and spread of the virus requires reverse transcription of the retroviral RNA to proviral DNA by the enzyme reverse transcriptase (RT). ${ }^{7,8}$ NNRTIs bind in a noncompetetive way to a unique region on the enzyme, namely nonsubstrate-binding site, resulting in alteration of its function and therefore achieving supression of HIV-1 replication with high selectivity. ${ }^{9}$ Although combined use of nucleoside inhibitors of RT (NRTIs), non-nucleoside RT inhibitors (NNRTIs) and protease inhibitors (PIs) in the treatment of HIV infections may provide a dramatic recovery in most HIV-infected persons, resistance to the currently used agents remains as a clinical problem. ${ }^{5}$ Consequenlty, there is still a need for novel and safe antiviral agents with potent and selective action which are also effective against mutant strains of HIV. Nevirapine, etravirine and efavirenz are representative examples of NNRTIs used in clinical practice today. ${ }^{10}$ Ongoing studies for discovery of new antiviral agents then gave rise to novel types NNRTIs which include tetrahydroimidazobenzodiazepinthione (TIBO) compounds ${ }^{11}$, phenethylthiazolylthiourea (PETT) derivatives $^{11}$, and $1 \mathrm{H}, 3 \mathrm{H}$-thiazolo[3,4-a]benzimidazoles (TBZs). ${ }^{12}$

Recent studies on molecular modification of the latter (TBZs) revealed that, dismantling of the imidazole nucleus leading to the design of new 1,3-thiazolidin-4-one derivatives, maintained the molecular requirements for enzyme inhibition. ${ }^{12}$

A literature search revealed that, 4-thiazolidinone derivatives may exhibit antibacterial ${ }^{13-14}$, antituberculosis, ${ }^{15-17}$ antiviral ${ }^{9,12,18-23}$ and anticancer ${ }^{24-27}$ properties. According to Andres et al. ${ }^{13}$, 4-thiazolidinones may be considered as phosphate bioisosteres and therefore inhibit the bacterial enzyme MurB which is involved in the biosynthesis of peptidoglycan layer of the cell wall. ${ }^{13}$ In 
addition, some thiazolidinones were recently reported as novel inhibitors of mycobacterial rhamnose synthetic enzymes. ${ }^{4}$ This new approach is believed to be selective as rhamnose which is not found in humans, has been shown to be essential for mycobacterial cell wall synthesis. ${ }^{4}$ Based on these observations, we have designed and synthesized a number of 2,3-disubstituted 1,3-thiazolidin-4-ones 8-11 as well as their 5-arylalkylidene derivatives 12-27. The present paper describes synthesis of four different derivatives of 1-[2-(Benzoylamino)-4-(methylthio)butyryl]4-alkyl/arylalkyl-thiosemicarbazides 4-7 and N-\{1-[[2-(3-alkyl/arylalkyl-4-oxo-1,3-thiazolidin2-ylidene)hydrazino]carbonyl]-3-(methylthio)-propyl benzamides 8-11; together with sixteen different derivatives of N-\{1-[[2-[5-(nonsubstituted/substituted benzylidene)-3-alkyl/arylalkyl-4oxo-1,3-thiazolidin-2-ylidene]-hydrazino]carbonyl]-3-(methyl-thio)propyl\} benzamide 12-27. Structures of the synthesized compounds were elucidated by the use of their UV, IR, ${ }^{1} \mathrm{H}-\mathrm{NMR}$, ${ }^{13} \mathrm{C}-\mathrm{NMR}$, HR-EI and HR-FAB Mass spectral data. The presence of the thiazolidinone pharmacophore as reported in previous studies, led us to investigate their antimicrobial, antimycobacterial and antiviral properties.

\section{Results and Discussion}

\section{Chemistry}

Synthetic route to 1-27 is presented in Scheme 1. $N$-Benzoyl methionine 1 was prepared through the Schotten Baumann reaction procedure. 2-(Benzoylamino)-4-(methylthio)butyric acid methyl ester 2 was obtained by the reaction of $N$-benzoyl methionine $\mathbf{1}$ and methanol in the presence of a few drops of concentrated sulfuric acid. 2-(Benzoylamino)-4-(methylthio)butyric acid hydrazide 3 was prepared by heating compound $\mathbf{2}$ with hydrazine hydrate in methanol. By condensing the hydrazide 3 with methyl, ethyl, allyl and benzyl isothiocyanates in ethanol, 1-[2(benzoylamino)-4-(methylthio)butyryl]-4-alkyl/arylalkyl thiosemicarbazides 4-7 were synthesized. $N$-[1-\{[2-(3-alkyl/arylalkyl-4-oxo-1,3-thiazolidin-2-ylidene)hydrazino]carbonyl $\}$-3(methylthio)propyl]benzamides 8-11 were synthesized by refluxing the thiosemicarbazides 4-7 mentioned above and ethylbromoacetate in the presence of sodium acetate in absolute ethanol. Finally, synthesis of $N$-\{1-[[2-[5-(nonsubstituted/substituted benzylidene)-3-alkyl/arylalkyl-4oxo-1,3-thiazolidin-2-ylidene]-hydrazino]carbonyl]-3-(methylthio)propyl $\}$ benzamide derivatives 12-27 were carried out by refluxing compounds 8-11 with aryl aldehydes in the presence of sodium methoxide in methanolic medium through Knoevenagel's reaction procedure .

The synthesized compounds were characterized by their IR, ${ }^{1} \mathrm{H}-\mathrm{NMR},{ }^{13} \mathrm{C}-\mathrm{NMR}$, HR-EI and HR-FAB Mass Spectral data. The IR spectra of compound 1 was characterized by the presence of a new $\mathrm{C}=\mathrm{O}$ absorption band at $1637 \mathrm{~cm}^{-1}$. IR spectral data of compound 2 was also confirmed on the basis of IR spectral data in literature. ${ }^{28}$ The band at $1650 \mathrm{~cm}^{-1}$ was attributed to the $\mathrm{C}=\mathrm{O}$ streching band of hydrazide. The bands at $3315 \mathrm{~cm}^{-1}$ and $3267 \mathrm{~cm}^{-1}$ were assigned to $\mathrm{NH}_{2}$ bands of hydrazide and they did not occur in the IR spectra of compounds 4-7. Absorption bands at $1212-1247 \mathrm{~cm}^{-1}$, which were attributed to the $\mathrm{C}=\mathrm{S}$ streching vibrations observed in the IR spectra 
of compounds 4-7. New $\mathrm{C}=\mathrm{O}$ bands $\left(1701-1725 \mathrm{~cm}^{-1}\right)$ in the IR spectra of 1,3-thiazolidine-4ones provided confirmatory evidence for ring closure. ${ }^{29-31}$ The Ar-Cl and Ar-F absorption bands were observed in the IR spectra of the compounds 12-27.

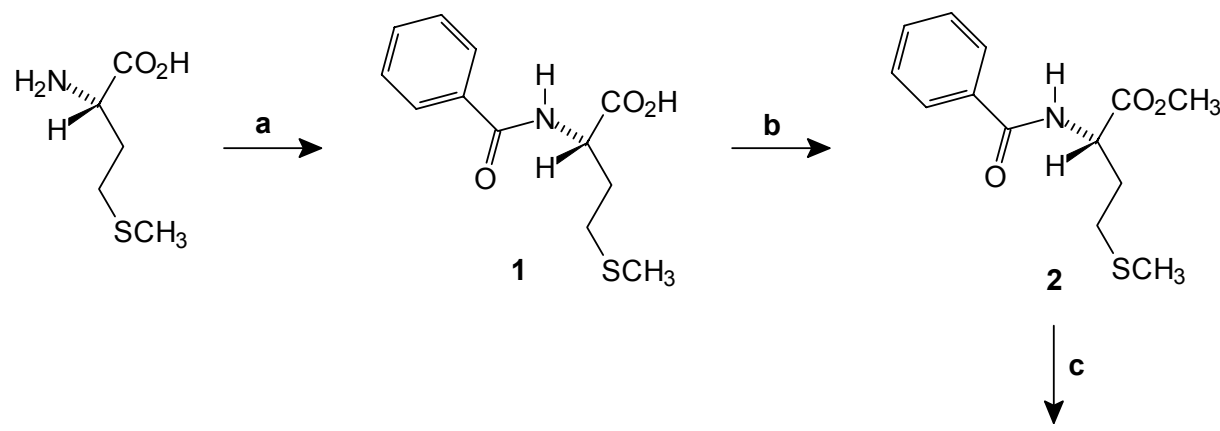<smiles>[R]NC(=S)NNC(=O)CCNC(=O)c1ccccc1</smiles>

4-7

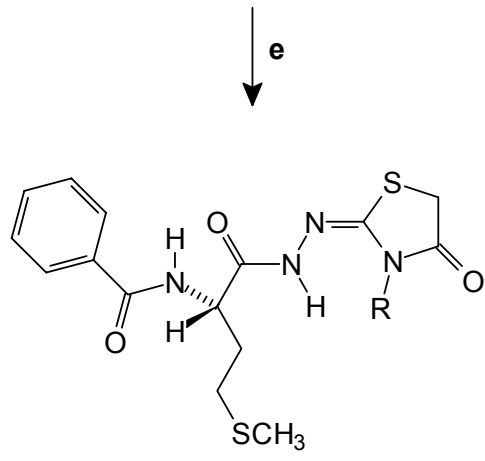

8-11
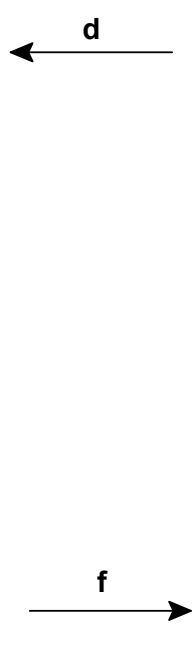

Scheme 1. Synthetic route to compounds 1-27. Reagents and conditions : (a) $\mathrm{C}_{6} \mathrm{H}_{5} \mathrm{COCl} /$ $\mathrm{NaOH}$; (b) $\mathrm{MeOH} / \mathrm{H}_{2} \mathrm{SO}_{4}$; (c) $\mathrm{NH}_{2} \mathrm{NH}_{2} . \mathrm{H}_{2} \mathrm{O}$; (d) R-NCS / EtOH, reflux ; (e) $\mathrm{Br}-\mathrm{CH}_{2}-$ $\mathrm{CO}_{2} \mathrm{C}_{2} \mathrm{H}_{5} / \mathrm{NaOAc} / \mathrm{EtOH}$, reflux ; (f) $\mathrm{Ar}-\mathrm{CHO} / \mathrm{NaOMe} / \mathrm{MeOH}$, reflux.

The exhibited chemical shifts obtained from ${ }^{1} \mathrm{H}-\mathrm{NMR}$ spectra of compounds 4-7 supported the proposed structures of the compounds. Resonances assigned to the $\mathrm{N}^{1}-\mathrm{H}, \mathrm{N}^{2}-\mathrm{H}, \mathrm{N}^{4}-\mathrm{H}$ protons of thiosemicarbazides 4-7 were detected at 10.05-10.12, 9.23-9.35, 7.47-8.94 ppm, respectively, which are supported by the literature. ${ }^{32-34}$ Other $\mathrm{NH}$ proton of thiosemicarbazides 4-7 which belong to benzoylamino group were detected at 7.68-8.94 ppm. Absence of resonances assigned to the $\mathrm{N}^{1}-\mathrm{H}, \mathrm{N}^{2}-\mathrm{H}, \mathrm{N}^{4}-\mathrm{H}$ protons of thiosemicarbazides 4-7 and the detected signals at about 
3.65-3.97 and 3.74-4.11 ppm attributed to the $\mathrm{CH}_{2}$ protons at the $5^{\text {th }}$ position of the 1,3thiazolidine-4-one ring supported the exact structures of 8-11. The endocyclic $-\mathrm{CH}_{2^{-}}$protons are used to be detected as a singlet peak with an integration of two protons but in our work they were detected as two singlet peaks with an integration of two protons. The $-\mathrm{NH}-\mathrm{N}=\mathrm{C}<$ protons of $1,3-$ thiazolidin-4-ones 8-11 were detected as two singlet peaks at 7.71-8.00 ppm and 9.15-9.35 ppm. The signals at 7.02-7.34 were attributed to Ar-CO-NH. ${ }^{1} \mathrm{H}-\mathrm{NMR}$ spectra of compounds 8-11 revealed the presence of two isomers as concluded from the $-\mathrm{CONHN},-\mathrm{H}_{3} \mathrm{C}-\mathrm{S}-\mathrm{CH}_{2}-\mathrm{CH}$, endocyclic $-\mathrm{CH}_{2}$ protons. As an appraisal the first singlet peak belongs to $\mathrm{E}$ isomer and the second one belongs to $\mathrm{Z}$ isomer and this may be explained on the basis of the difference in the relative stability of $\mathrm{E}$ and $\mathrm{Z}$ isomers formed due to the rotational restriction about the exocyclic $\mathrm{N}=\mathrm{C}$ bond. ${ }^{35}$ Compound $\mathbf{8}$ was selected as prototype and its ${ }^{13} \mathrm{C}-\mathrm{NMR}$ spectrum was used for further support. Detecting $-\mathrm{H}_{3} \mathrm{C}-\mathrm{S}-\mathbf{C H}_{2}-\mathrm{CH},-\mathrm{H}_{3} \mathrm{C}-\mathrm{S}-\mathrm{CH}_{2}-\boldsymbol{C H}$, endocyclic $-\boldsymbol{C H}_{2}$, both of the $\mathbf{C}=\mathrm{O}$ and some of the aromatic $\mathrm{C}$ atoms as two peaks in stead of one provided confirmatory evidence for geometric isomerism.

The representative example compound $\mathbf{8}$ was fragmented via three prominent pathways to afford fragments at m/z 306.0809, m/z 145.0303 and m/z 232.0955 based on thioether moiety, $\mathrm{CONH}$ bond cleavage, benzoyl cation as base peak and methylidene sulfonium cation, respectively, in accordance with literature. ${ }^{35,36}$ Compounds 9-11 were fragmented via quasimolecular ion by HR-FAB ${ }^{37}$.

The purity of compounds 4-27 was demonstrated by TLC and HPLC. There were no spots or peaks accounting for the starting compounds; but for some compounds we observed more than one peak with the same UV spectra. By using HPLC-UV/DAD peak homogeneity was proved.

Resonances assigned to the endocyclic $-\mathrm{CH}_{2}$ - protons at the $5^{\text {th }}$ position of the 1,3 -thiazolidine-4one ring were not detected and instead of the mentioned proton's signal, two peaks with an integration of one proton symbolizing the methine proton of $>\mathrm{C}=\mathrm{CH}$-Ar moiety was observed between 7.23-8.11 ppm. ${ }^{38}$ Considering the aromatic region of ${ }^{1} \mathrm{H}-\mathrm{NMR}$ spectra of compounds 12-27 displayed signals of aromatic protons of arylidene segment as an evidence for the supposed structures. For further evidence to prove the geometric isomerism as a reason of rotational restriction of $-\mathrm{N}=\mathrm{C}<$ and $>\mathrm{C}=\mathrm{CH}-\mathrm{Ar}$ bonds ${ }^{13} \mathrm{C}-\mathrm{NMR}$ spectra of compound $\mathbf{1 3}$ was observed. ${ }^{13} \mathrm{C}-\mathrm{NMR}$ data of the representative compounds 8 and $\mathbf{1 3}$, which were obtained using DEPT technique at $100 \mathrm{MHz}$, have also supported the carbon framework by discrimination of $\mathrm{CH}_{3}, \mathrm{CH}_{2}, \mathrm{CH}$ and quarternary carbons $\left(\mathrm{C}_{\mathrm{q}}\right)$ (see experimental section). On the other hand, high accuracy between experimental and calculated ${ }^{13} \mathrm{C}$ chemical shifts were also observed (Table 1). Calculations of the ${ }^{13} \mathrm{C}$-NMR chemical shifts were performed using ACD/CNMR Predictor software available online at ACD/I-Lab Interactive Laboratory website (http://www.acdlabs.com/ilab).

Resonances due to the $\mathrm{R}_{1}$ and $\mathrm{R}_{2}$ groups of the compounds were recorded at expected values. The benzylic protons of compound 11 and compounds 23-27 were observed at 5.01-5.07 ppm and 4.80-5.03 ppm, respectively, and the benzylic protons of compound $\mathbf{1 1}$ could easily be differentiated from endocyclic $\mathrm{CH}_{2}$ protons at the $5^{\text {th }}$ position of the 1,3-thiazolidine-4-one ring. 
The representative example compound 14 fragmented via two prominent pathways to afford fragments by $-\mathrm{CONH}$ bond cleavage followed by dissociation of the thioether moiety and -NHN bond cleavage.

Table 1. Assignment of ${ }^{13} \mathrm{C}$ NMR spectra of compounds 4,8 and $13^{\mathrm{a}}$

\begin{tabular}{|c|c|c|c|c|c|c|}
\hline O & $\overbrace{}^{n-5} \int^{H}$ & 21 & $\sum_{6}^{\mathrm{SCH}_{7}}$ & $12 \underbrace{11}$ & 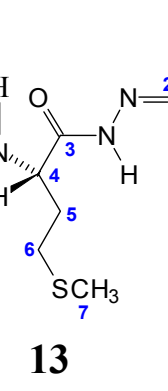 & $\overbrace{0}^{16} \overbrace{22}^{17} \overbrace{21}^{19}$ \\
\hline Assignment & \multicolumn{2}{|c|}{$\begin{array}{c}\text { Compound } \mathbf{4} \\
\delta \mathbf{C}(\mathrm{ppm})\end{array}$} & \multicolumn{2}{|c|}{$\begin{array}{c}\text { Compound } \mathbf{8} \\
\delta \mathbf{C}(\mathrm{ppm})\end{array}$} & \multicolumn{2}{|c|}{$\begin{array}{c}\text { Compound } \mathbf{1 3} \\
\delta \mathbf{C}(\mathrm{ppm})\end{array}$} \\
\hline Carbon \# & calculated & found & calculated & found & calculated & found \\
\hline 1 & 30.8 & 31.3 & 29.1 & 29.6 & 30.3 & $29.9 / 30.1$ \\
\hline 2 & 175.7 & 182.7 & 160.8 & 159.8 & 160.7 & 152.6 \\
\hline 3 & 165.3 & 167.9 & 164.7 & $\begin{array}{c}167.0 / 167.2 / \\
168.5\end{array}$ & 164.7 & 165.5 \\
\hline 4 & 52.8 & 52.7 & 56.1 & 52.4 & 56.1 & 52.4 \\
\hline 5 & 31.6 & 30.8 & 31,0 & 31.8 & 31,0 & 31.6 \\
\hline 6 & 29.5 & 30.2 & 29.7 & 30.5 & 29.7 & 30.5 \\
\hline 7 & 15.8 & 15.1 & 15.3 & 15.2 & 15.3 & 15.2 \\
\hline 8 & 167.9 & 172.5 & 170.6 & $171.3 / 171.9$ & 170.6 & 168.8 \\
\hline 9 & 133.2 & 134.0 & 135.5 & $134.4 / 134.6$ & 135.5 & 134.4 \\
\hline 10,14 & 127.0 & 128.1 & 127.9 & 127.9 & 127.9 & $127.9 / 128.1$ \\
\hline 11,13 & 128.3 & 128.7 & 128.4 & $128.1 / 128.6$ & 128.4 & 128.6 \\
\hline 12 & 131.70 & 132.1 & 131.6 & 131.8 & 131.6 & 129.7 \\
\hline 15 & - & - & 171.3 & 173.5 & 168.2 & 167.2 \\
\hline 16 & - & - & 33.7 & 33.3 & 131.7 & 135.1 \\
\hline 17 & - & - & - & - & 145.7 & 131.8 \\
\hline 18 & - & - & - & - & 130.6 & 131.9 \\
\hline 19,23 & - & - & - & - & 132.3 & 131.7 \\
\hline 20,22 & - & - & - & - & 129.2 & 128.7 \\
\hline 21 & - & - & - & - & 133.5 & 132.7 \\
\hline
\end{tabular}

${ }^{\mathrm{a}}$ Calculation of the ${ }^{13} \mathrm{C}$ NMR chemical shifts were performed using ACD/CNMR Predictor software available online at ACD/I-Lab Interactive Laboratory website (http://www.acdlabs.com/ilab). See experimental section for details of ${ }^{13} \mathrm{C}$ chemical shifts obtained using DEPT technique. 


\section{Biological Activity}

In view of the antimycobacterial and antiviral activity ascertained for similar 4-thiazolidinones, the synthesized compounds were evaluated for their antibacterial, antituberculosis and antiviral effects. Compounds 24-26 exhibited marginal activity (MIC $=250 \mu \mathrm{g} / \mathrm{ml}$ ) against Staphylococcus aureus ATCC 29213, Bacillus subtilis A57 and Candida albicans A177. Ofloxacin, sulbactam + cefoperazone and/or netilmicin were used as positive reference standards to determine the sensitivity of one strain/isolate in each microbial species tested.

In vitro evaluation of antimycobacterial activity against M.tuberculosis H37Rv was carried out at the Tuberculosis Antimicrobial Acquisition and Coordinating Facility (TAACF), National Institute of Allergy and Infectious Diseases, Southern Research Institute, Birmingham, Alabama, USA. Primary screen was conducted for all of the synthesized compounds 1-27 at $6.25 \mu \mathrm{g} / \mathrm{ml}$ against M.tuberculosis H37Rv in BACTEC 12B medium using the BACTEC 460 radiometric system. Rifampicin was used as the standard in the anti-tuberculosis activity screening. The most active derivative was identified as 27 with $90 \%$ inhibition of mycobacterial growth at 6.25 $\mu \mathrm{g} / \mathrm{ml}$. The remaining compounds showed marginal or no effect at this concentration.

Antiviral activities of the synthesized compounds were screened against various types of viruses in HEL, HeLa and Vero cell cultures. Anti-HIV and cytotoxicity data were also obtained with the compounds using the strains HIV-1(III $\left.\mathrm{B}_{\mathrm{B}}\right)$ and HIV-2(ROD) in an MT-4/MTT based assay. The compounds were also evaluated for in vitro antiviral activity against herpes simplex virus [HSV-1 (strain KOS), thymidine kinase deficient $\left(\mathrm{TK}^{-}\right)$strain of $\mathrm{HSV}-1$ resistant to acyclovir $\left(\mathrm{ACV}^{\mathrm{R}}\right)$, HSV-2 $\left.(\mathrm{G})\right]$, vaccinia virus (VV) and vesicular stomatitis virus (VSV) in HEL cell cultures ; VSV, Coxsackie virus B4 and respiratory syncytial virus (RSV) in HeLa cell cultures ; Parainfluenza-3 virus, Reovirus-1, Sindbis virus, Coxsackie virus B4 and Punta Toro virus in Vero cell cultures. Acyclovir, Brivudin, (S)-DHPA, Ganciclovir and Ribavirin were used as standard drugs for comparison of the test compounds. For anti-HIV screening, nevirapine, delaviridine, efavirenz and zidovudine were used as the reference drugs. None of the tested compounds showed antiviral activity at subtoxic doses whereas some of them exhibited remarkable cytotoxic potential.

Compounds 8, 13, 14, 23, 25 were evaluated for their anticancer activity against 60 human tumoral cell lines derived from nine different cancer types (non-small cell lung, colon, breast, ovarian, renal, prostate, CNS, leukemia and melanoma) by the National Cancer Institute (NCI). They did not exhibit anticancer activity having $\mathrm{GI}_{50}$ values at a high concentration. Therefore, these compounds were not selected for further testing.

\section{Experimental Section}

General Procedures. All melting points $\left({ }^{\circ} \mathrm{C}\right.$, uncorrected) were determined using Büchi 530 melting point apparatus. Infrared spectra were recorded in $\mathrm{KBr}$ disc using BIO-RAD FTS-135, FT-IR spectrometer and expressed in wavenumber $v\left(\mathrm{~cm}^{-1}\right)$. NMR spectra were obtained on a 
Bruker AVANC-DPX $400 \mathrm{NMR}$ spectrometer at $400 \mathrm{MHz}$ for ${ }^{1} \mathrm{H}$ and $100 \mathrm{MHz}$ for ${ }^{13} \mathrm{C}-\mathrm{DEPT}$, the chemical shifts are expressed in $\delta(\mathrm{ppm})$ downfield from tetramethylsilane (TMS) using $\mathrm{CDCl}_{3}, \mathrm{CDCl}_{3}$-acetone and $\mathrm{CDCl}_{3}$-DMSO as solvent. High resolution electron impact and fast atom bombardment mass spectra were recorded on a Jeol JMS-700 instrument. The liquid chromatographic system, used in the present study, consisted of an Agilent technologies 1100 series instrument equipped with a quaternary solvent delivery system and a model Agilent series G-1315 A photodiode array detector. A Rheodyne syringe loading sample injector with a $50 \mu 1$ sample loop was used for the injection of analytes. Chromatographic data were collected and processed using Agilent Chemstation Plus software. The separation was performed at ambient temperature, on a reversed phase NovoPak - C18 column $(150 \times 3.9 \mathrm{~mm} ; 5 \mu \mathrm{m}$ particle size $)$. All experiments were employed in the isocratic mode. The mobile phase was prepared by mixing methanol, acetonitrile and distilled water $(50: 10: 40, \mathrm{v} / \mathrm{v} / \mathrm{v})$. This phase was filtered through a $0.45 \mu \mathrm{m}$ membrane and degassed by ultrasonication, prior to use. Solvent delivery was employed at a flow rate of $1.0 \mathrm{ml} \cdot \mathrm{min}^{-1}$. Detection of the analytes was carried out at $210 \mathrm{~nm}$.

2-(Benzoylamino)-4-(methylthio)butyric acid (1). 2-Amino-4-(methylthio)butyric acid (1.492 $\mathrm{g}, 0.01 \mathrm{~mol})$ was dissolved in sodium hydroxide solution $(100 \mathrm{ml}, 0.02 \mathrm{~mol})$ and benzoyl chloride $(1.405 \mathrm{~g}, 0.01 \mathrm{~mol})$ was added to the reaction medium with stirring in ice bath. The crude product was precipitated by using conc. $\mathrm{HCl}$, filtered and washed with water, dried and crystallized from ethanol- $\mathrm{H}_{2} \mathrm{O}$. The product was washed with boiling petroleum ether. Yield 84\%. M.p. 88-90 ${ }^{\circ} \mathrm{C}$ (lit. 95-96 $\left.{ }^{\circ} \mathrm{C}\right)^{39}$. IR, v $\left(\mathrm{cm}^{-1}\right)$ : 3404 (w), 3323 (br), 3063, 2973, 2837 (w), 1755 (m), 1637 (s), 1576 (m), 1543 (s), 1487 (w), 1446 (m), 1259 (w), 688 (m), 644 (w). HR-MS $\left(\mathrm{EI}^{+}\right), \mathrm{m} / \mathrm{z}: 253.0763\left(\mathrm{M}^{+}, \mathrm{C}_{12} \mathrm{H}_{15} \mathrm{NO}_{3} \mathrm{~S}\right.$ requires 253.0774$), 192.0667\left(\mathrm{C}_{10} \mathrm{H}_{10} \mathrm{NO}_{3}\right), 179.0576$ $\left(\mathrm{C}_{9} \mathrm{H}_{9} \mathrm{NO}_{3}\right), 161.0466\left(\mathrm{C}_{9} \mathrm{H}_{7} \mathrm{NO}_{2}\right), 105.0344\left(\mathrm{C}_{7} \mathrm{H}_{5} \mathrm{O}\right), 77.0393\left(\mathrm{C}_{6} \mathrm{H}_{5}\right)$.

2-(Benzoylamino)-4-(methylthio) butyric acid methyl ester (2). 2-(Benzoylamino)-4(methylthio)butyric acid $(2.53 \mathrm{~g}, 0.01 \mathrm{~mol})$ was dissolved in $20 \mathrm{ml}$ methanol and $1 \mathrm{ml}$ conc. $\mathrm{H}_{2} \mathrm{SO}_{4}$ was added. The reaction mixture was heated under reflux for $2 \mathrm{~h}$. The crude product was precipitated by using $\mathrm{NaHCO}_{3}$ solution (5\%), filtered, dried and washed with boiling petroleum ether. Yield $68 \%$. M.p. $86-88^{\circ} \mathrm{C}$ (lit. $87.5-88^{\circ} \mathrm{C}$, crystallized from methanol). ${ }^{40}$ IR, v $\left(\mathbf{c m}^{-1}\right)$ : 3311 (br), 3069, 2957, 2923, 2836 (w), 1759, 1639 (vs), 1582 (m), 1532 (s), 1491, 1439, 1269 (m), 1217, 1171(s), 1096 (m), 693 (s). HR-MS (EI $\left.{ }^{+}\right), \mathbf{m} / \mathbf{z}: 267.0927\left(\mathrm{M}^{+}, \mathrm{C}_{13} \mathrm{H}_{17} \mathrm{NO}_{3} \mathrm{~S}\right.$ requires 267.0930), $206.0832\left(\mathrm{C}_{11} \mathrm{H}_{12} \mathrm{NO}_{3}\right), 193.0739\left(\mathrm{C}_{10} \mathrm{H}_{11} \mathrm{NO}_{3}\right), 161.0455\left(\mathrm{C}_{9} \mathrm{H}_{7} \mathrm{NO}_{2}\right)$, $105.0331\left(\mathrm{C}_{7} \mathrm{H}_{5} \mathrm{O}\right), 77.0400\left(\mathrm{C}_{6} \mathrm{H}_{5}\right)$.

2-(Benzoylamino)-4-(methylthio) butyric acid hydrazide (3). 2-(Benzoylamino)-4(methylthio) butyric acid methyl ester (2) $(2.67 \mathrm{~g}, 0.01 \mathrm{~mol})$ and hydrazine hydrate $(80 \%, 3.1275$ $\mathrm{g}, 0.05 \mathrm{~mol}$ ) was heated under reflux for $1 \mathrm{~h}$ and $25 \mathrm{ml}$ methanol was added to the reaction medium and again it was heated under reflux for $1 \mathrm{~h}$. The crude product was filtered and crystallized from methanol. Yield 59\%. M.p. $180^{\circ} \mathrm{C}$. IR, v (cm $\left.{ }^{-1}\right)$ : 3315, 3267 (br), 3078, 2976, 2917 (w), 1635, 1650, 1578, 1547, 1493 (s), 1448, 1427 (w), 1273,1250, 1205 (m), 700, 670 (m). HR-MS (EI $\left.{ }^{+}\right), \mathbf{m} / \mathbf{z}: 267.1026\left(\mathrm{M}^{+}, \mathrm{C}_{12} \mathrm{H}_{17} \mathrm{~N}_{3} \mathrm{O}_{2} \mathrm{~S}\right.$ requires 267.1043$), 236.0740\left(\mathrm{C}_{12} \mathrm{H}_{14} \mathrm{NO}_{2} \mathrm{~S}\right)$, 
$208.0785\left(\mathrm{C}_{11} \mathrm{H}_{14} \mathrm{NOS}\right), \quad 193.0851\left(\mathrm{C}_{9} \mathrm{H}_{11} \mathrm{~N}_{3} \mathrm{O}_{2}\right), 162.0531\left(\mathrm{C}_{9} \mathrm{H}_{8} \mathrm{NO}_{2}\right), 160.0746\left(\mathrm{C}_{10} \mathrm{H}_{10} \mathrm{NO}\right)$, $105.0345\left(\mathrm{C}_{7} \mathrm{H}_{5} \mathrm{O}\right), 77.0395\left(\mathrm{C}_{6} \mathrm{H}_{5}\right), 61.0128\left(\mathrm{C}_{2} \mathrm{H}_{5} \mathrm{~S}\right)$.

1-[2-(Benzoylamino)-4-(methylthio) butyryl]-4-alkyl/arylalkyl thiosemicarbazides (4-7). 2(Benzoylamino)-4-(methylthio) butyric acid hydrazide (3) was heated with appropriate isothiocyanates under reflux for $2 \mathrm{~h}$ in ethanol.The crude products (4-7) were filtered and crystallized from ethanol.

1-[2-(Benzoylamino)-4-(methylthio) butyryl]-4-methyl thiosemicarbazide (4). Compound 4 was synthesized by the reaction of compound $3(2.67 \mathrm{~g}, 0.01 \mathrm{~mol})$ with methyl isothiocyanate (98\%, 0.745g, $0.01 \mathrm{~mol})$. Yield 97\%. M.p. $188^{\circ} \mathrm{C}$. HPLC $\mathrm{t}_{\mathrm{R}}(\mathrm{min}): 1.762 . \mathbf{I R ,} \mathbf{v}\left(\mathbf{c m}^{-1}\right): 3316$, 3216 (br), 3063, 2973, 2837 (w), 1697, 1641 (s), 1561, 1529 (s), 1489, 1437, 1296, 1212 (m). ${ }^{1} \mathbf{H}$ NMR : $\boldsymbol{\delta}(\mathbf{p p m}) 2.58(\mathrm{t}, 2 \mathrm{H}, J=7.1 \mathrm{~Hz}, J=7.1 \mathrm{~Hz}), 2.97$ (d, 3H, $J=4.2 \mathrm{~Hz}), 4.46-4.64$ (q, 1H, $J$ $=7.0 \mathrm{~Hz}), 7.33$ (t, $2 \mathrm{H}, J=7.3 \mathrm{~Hz}, J=8.0 \mathrm{~Hz}), 7.43(\mathrm{t}, 1 \mathrm{H}, J=7.3 \mathrm{~Hz}, J=7.5 \mathrm{~Hz}), 7.47-7.63$ $(\mathrm{s}, 1 \mathrm{H}), 7.77(\mathrm{~d}, 2 \mathrm{H}, J=7.3 \mathrm{~Hz}), 7.85(\mathrm{~d}, 1 \mathrm{H}, J=5,9 \mathrm{~Hz}), 7.99(\mathrm{~s}, 1 \mathrm{H}), 9.28(\mathrm{~s}, 1 \mathrm{H}) .{ }^{13} \mathbf{C}$ NMRDEPT : $\boldsymbol{\delta}$ (ppm) $15.1\left(\mathrm{CH}_{3}\right), 30.2\left(\mathrm{CH}_{2}\right), 30.8\left(\mathrm{CH}_{2}\right), 31.3\left(\mathrm{CH}_{3}\right), 52.7(\mathrm{CH}), 128.1(\mathrm{CH}), 128.7$ $(\mathrm{CH}), 132.1(\mathrm{CH}), 134.0\left(\mathrm{C}_{\mathrm{q}}\right), 167.9\left(\mathrm{C}_{\mathrm{q}}\right), 172.5\left(\mathrm{C}_{\mathrm{q}}\right), 182.7\left(\mathrm{C}_{\mathrm{q}}\right)$. HR-MS $\left(\mathbf{E I}^{+}\right), \mathbf{m} / \mathbf{z}: 340.1022$ $\left(\mathrm{M}^{+}, \mathrm{C}_{14} \mathrm{H}_{20} \mathrm{~N}_{4} \mathrm{O}_{2} \mathrm{~S}_{2}\right.$ requires 340.1030), $322.0919\left(\mathrm{C}_{14} \mathrm{H}_{18} \mathrm{~N}_{4} \mathrm{OS}_{2}\right), 248.0731\left(\mathrm{C}_{11} \mathrm{H}_{12} \mathrm{~N}_{4} \mathrm{OS}\right)$, 236.0708 $\left(\mathrm{C}_{12} \mathrm{H}_{14} \mathrm{NO}_{2} \mathrm{~S}\right), 208.0778\left(\mathrm{C}_{11} \mathrm{H}_{14} \mathrm{NOS}\right), 193.0869\left(\mathrm{C}_{9} \mathrm{H}_{11} \mathrm{~N}_{3} \mathrm{O}_{2}\right), 162.0529\left(\mathrm{C}_{9} \mathrm{H}_{8} \mathrm{NO}_{2}\right)$, 160.0736 $\left(\mathrm{C}_{10} \mathrm{H}_{10} \mathrm{NO}\right), 105.0337\left(\mathrm{C}_{7} \mathrm{H}_{5} \mathrm{O}\right), 77.0400\left(\mathrm{C}_{6} \mathrm{H}_{5}\right), 72.9974\left(\mathrm{C}_{2} \mathrm{H}_{3} \mathrm{NS}\right)$.

1-[2-(Benzoylamino)-4-(methylthio) butyryl]-4-ethyl thiosemicarbazide (5). Compound 5 was synthesized by the reaction of compound $3(2.67 \mathrm{~g}, 0.01 \mathrm{~mol})$ with ethyl isothiocyanate (0.871 g, $0.01 \mathrm{~mol})$. Yield 70\%. M.p. $168^{\circ} \mathrm{C}$. HPLC $\mathrm{t}_{\mathrm{R}}(\mathrm{min}): 2.005$. IR, v (cm $\left.{ }^{-1}\right): 3246,3215$, 3176 (br), 3067, 2972 (w), 1704 (s), 1637 (vs), 1579 (w), 1541(s), 1491 (m), 1439 (w), 1314 (m), $1228(\mathrm{w}) .{ }^{1} \mathbf{H}$ NMR : $\boldsymbol{\delta}$ (ppm) 1.09 ( t, 3H, $J=7.2 \mathrm{~Hz}, J=7.2 \mathrm{~Hz}$ ), 1.97-2.03 (q, 5H), 2.45-2.54 (m, 2H), 3.42-3.47 (m ,2H), $4.28(\mathrm{~s}, 1 \mathrm{H}), 7.31$ and 7,39 (2t, 2H, J=7.3 Hz, $J=7.6$ $\mathrm{Hz}$ and $J=7.3 \mathrm{~Hz}, J=7.3 \mathrm{~Hz}), 7.50(\mathrm{t}, J=4.9 \mathrm{~Hz}, J=4.6 \mathrm{~Hz}), 7.68(\mathrm{~s}, 1 \mathrm{H}), 7.80(\mathrm{~d}, 2 \mathrm{H}, J=8.1$ $\mathrm{Hz}), 8.54(\mathrm{~s}, 1 \mathrm{H}), 8.82(\mathrm{~s}, 1 \mathrm{H}), 9.99(\mathrm{~s}, 1 \mathrm{H}) . \mathbf{H R}-\mathbf{M S}\left(\mathbf{E I ^ { + }}\right), \mathbf{m} / \mathbf{z}: 354.1210\left(\mathrm{M}^{+}, \mathrm{C}_{15} \mathrm{H}_{22} \mathrm{~N}_{4} \mathrm{O}_{2} \mathrm{~S}_{2}\right.$ requires 354.1186), $336.1056\left(\mathrm{C}_{15} \mathrm{H}_{20} \mathrm{~N}_{4} \mathrm{O}_{2} \mathrm{~S}\right), 259.1154\left(\mathrm{C}_{13} \mathrm{H}_{15} \mathrm{~N}_{4} \mathrm{O}_{2}\right), 246.1110\left(\mathrm{C}_{12} \mathrm{H}_{14} \mathrm{~N}_{4} \mathrm{O}_{2}\right)$, 236.0739 $\left(\mathrm{C}_{12} \mathrm{H}_{14} \mathrm{NO}_{2} \mathrm{~S}\right), 141.0782\left(\mathrm{C}_{5} \mathrm{H}_{9} \mathrm{~N}_{4} \mathrm{O}\right), 105.0325\left(\mathrm{C}_{7} \mathrm{H}_{5} \mathrm{O}\right), 77.0416\left(\mathrm{C}_{6} \mathrm{H}_{5}\right)$.

1-[2-(Benzoylamino)-4-(methylthio) butyryl]-4-allyl thiosemicarbazide (6). Compound 6 was synthesized by the reaction of compound $3(2.67 \mathrm{~g}, 0.01 \mathrm{~mol})$ with allyl isothiocyanate $(95 \%$,

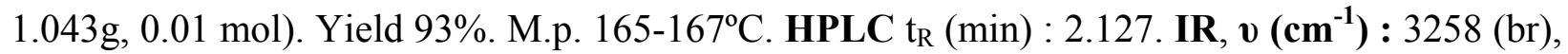
3063, 2977, 2917 (w), 1705, 1634, 1541(vs), 1485 (s), 1227 (w). ${ }^{1}$ H NMR : $\delta$ (ppm) 1.97-2.04 (q, 5H) 2.28-2.69 (m, 2H), $4.11(\mathrm{~s}, 2 \mathrm{H}), 4.34(\mathrm{~s}, 1 \mathrm{H}), 4.95$ (d, 1H, cis, J=10.3 Hz), 5.09 (d, 1H, trans, $J=17.2 \mathrm{~Hz}), 5.60-5.92(\mathrm{~m}, 1 \mathrm{H}), 7.29$ and $7.38(2 \mathrm{t}, 2 \mathrm{H}, J=7.6 \mathrm{~Hz}, J=7.8 \mathrm{~Hz}$ and $J=7.4$ $\mathrm{Hz}, J=7.1 \mathrm{~Hz}), 7.64(2 \mathrm{~s}, 1 \mathrm{H}), 7.77$ (d, 2H, $J=7.6 \mathrm{~Hz}), 8.48(\mathrm{~s}, 1 \mathrm{H}), 8.94(\mathrm{~s}, 1 \mathrm{H}), 9.35$ (s, 1H), $10.02(\mathrm{~s}, 1 \mathrm{H})$. HR-MS $\left(\mathbf{F A B}^{+}\right), \mathbf{m} / \mathbf{z}: 389.1068\left(\mathrm{M}+\mathrm{Na}, \mathrm{C}_{16} \mathrm{H}_{22} \mathrm{~N}_{4} \mathrm{O}_{2} \mathrm{~S}_{2} \mathrm{Na}\right), 367.1280(\mathrm{M}+\mathrm{H}$, $\left.\mathrm{C}_{16} \mathrm{H}_{23} \mathrm{~N}_{4} \mathrm{O}_{2} \mathrm{~S}_{2}\right)$.

1-[2-(Benzoylamino)-4-(methylthio) butyryl]-4-benzyl thiosemicarbazide (7). Compound 7 was synthesized by the reaction of compound 3 (2.67 g, $0.01 \mathrm{~mol})$ with benzyl isothiocyanate (97\%, 1.538g, $0.01 \mathrm{~mol})$. Yield 84\%. M.p. 177-182 ${ }^{\circ}$ C. HPLC $t_{R}(\min ): 2.945 . \mathbf{I R}, \mathbf{v}\left(\mathbf{c m}^{-1}\right)$ : 
3286, 3202 (br), 3058, 2967 (w), 1700, 1636, 1559 (vs), 1508, 1490, (m), 1457 (w), 1247 (w).

${ }^{1} \mathbf{H}$ NMR : $\boldsymbol{\delta}$ (ppm) 1.96-2.03 (p, 5H), 2.44-2.53 (m, 2H), 4.32 (s, 1H), 4.73 (t, 2H, $J=6.1 \mathrm{~Hz}, J$ $=6.4 \mathrm{~Hz}), 7.04-7.71(\mathrm{~m}, 10 \mathrm{H}), 8.09(\mathrm{~s}, 1 \mathrm{H}), 8.53(\mathrm{~s}, 1 \mathrm{H}), 9.05(\mathrm{~s}, 1 \mathrm{H}), 10.08(\mathrm{~s}, 1 \mathrm{H})$. HR-MS $\left(\mathbf{F A B}^{+}\right), \mathbf{m} / \mathbf{z}: 439.1238\left(\mathrm{M}+\mathrm{Na}, \mathrm{C}_{20} \mathrm{H}_{24} \mathrm{~N}_{4} \mathrm{O}_{2} \mathrm{~S}_{2} \mathrm{Na}\right), 417.1442\left(\mathrm{M}+\mathrm{H}, \mathrm{C}_{20} \mathrm{H}_{25} \mathrm{~N}_{4} \mathrm{O}_{2} \mathrm{~S}_{2}\right)$.

N-\{1-[[2-(3-Alkyl/arylalkyl-4-oxo-1,3-thiazolidin-2-ylidene)hydrazino]-carbonyl]-3-(methylthio)propyl $\}$ benzamides (8-11). A mixture of appropriate thiosemicarbazide (4-7), anhydrous sodium acetate $(99 \%, 3.32 \mathrm{~g}, 0.04 \mathrm{~mol})$ and ethyl bromoacetate $(1.837 \mathrm{~g}, 0.011 \mathrm{~mol})$ in $20 \mathrm{ml}$ absolute ethanol were heated under reflux for $2 \mathrm{~h}$. The mixture was cooled and the crude products (8-11) were filtered, dried and crystallized from ethanol.

\section{N-\{1-[[2-(3-Methyl-4-oxo-1,3-thiazolidin-2-ylidene)hydrazino]carbonyl]-3-(methylthio)-}

propyl benzamide (8). Compound 8 was synthesized according to the procedure given above for compounds 8-11 by using compound 4 (3.40 g, $0.01 \mathrm{~mol})$ as starting material. Yield 80\%. M.p. $208^{\circ} \mathrm{C}$. HPLC $t_{R}(\mathrm{~min})$ : 2.096. IR, v (cm $\left.{ }^{-1}\right)$ : 3263 (br), 3058, 3004, 2915 (w), 1725 (vs), 1668 (m), 1639, 1605 (s), 1534, 1534, 1427, 1365, 1323, 1231 (m). ${ }^{\mathbf{1}} \mathbf{H}$ NMR : $\delta$ (ppm) 2.04$2.27(\mathrm{~m}, 5 \mathrm{H}), 2.60$ and $2.78(\mathrm{~s}$ and $\mathrm{s}, 2 \mathrm{H}), 3.23$ and $3.24(\mathrm{~s}$ and $\mathrm{s}, 3 \mathrm{H}), 3.83(\mathrm{~s}, 1 \mathrm{H}), 3.90(\mathrm{~s}, 1 \mathrm{H})$, 4.85-4.87 and 5.39-5.62 (q and $\mathrm{m}, 1 \mathrm{H}), 7.11(\mathrm{~d}, 1 \mathrm{H}, J=7.6 \mathrm{~Hz}), 7.41(\mathrm{t}, 2 \mathrm{H}, J=7.2 \mathrm{~Hz}, J=7.7$ $\mathrm{Hz}$ ), 7.46-7.52 (q, 1H ), 7.76-7.81 (q, 2H), 7.71 and 9.15 (s and s, 1H). ${ }^{13}$ C NMR - DEPT: $\boldsymbol{\delta}$ (ppm) $15.2\left(\mathrm{CH}_{3}\right), 29.6\left(\mathrm{CH}_{3}\right), 30.5\left(\mathrm{CH}_{2}\right), 31.8\left(\mathrm{CH}_{2}\right), 33.3\left(\mathrm{CH}_{2}\right), 52.4(\mathrm{CH}), 127.9(\mathrm{CH})$, 128.1, $128.6(\mathrm{CH}), 131.8(\mathrm{CH}), 134.4,134.6\left(\mathrm{C}_{\mathrm{q}}\right), 159.8\left(\mathrm{C}_{\mathrm{q}}\right), 167.0,167.2,168.5\left(\mathrm{C}_{\mathrm{q}}\right), 171.3$, $171.9\left(\mathrm{C}_{\mathrm{q}}\right), 173.5\left(\mathrm{C}_{\mathrm{q}}\right)$. HR-MS (EI $\left.{ }^{+}\right), \mathbf{m} / \mathbf{z}: 380.0989\left(\mathrm{M}^{+}, \mathrm{C}_{16} \mathrm{H}_{20} \mathrm{~N}_{4} \mathrm{O}_{3} \mathrm{~S}_{2}\right.$ requires 380.0979), $306.0809 \quad\left(\mathrm{C}_{13} \mathrm{H}_{14} \mathrm{~N}_{4} \mathrm{O}_{3} \mathrm{~S}\right), \quad 236.0728 \quad\left(\mathrm{C}_{12} \mathrm{H}_{14} \mathrm{NO}_{2} \mathrm{~S}\right), \quad 232.0955 \quad\left(\mathrm{C}_{8} \mathrm{H}_{16} \mathrm{~N}_{4} \mathrm{O}_{2} \mathrm{~S}\right), \quad 208.0790$ $\left(\mathrm{C}_{11} \mathrm{H}_{14} \mathrm{NOS}\right), 145.0303\left(\mathrm{C}_{4} \mathrm{H}_{7} \mathrm{~N}_{3} \mathrm{O}\right), 105.0337\left(\mathrm{C}_{7} \mathrm{H}_{5} 0\right), 77.0391\left(\mathrm{C}_{6} \mathrm{H}_{5}\right)$.

\section{N-\{1-[[2-(3-Ethyl-4-oxo-1,3-thiazolidin-2-ylidene)hydrazino]carbonyl]-3-(methylthio)-}

propyl benzamide (9). Compound 9 was synthesized according to the procedure given above for compounds 8-11 by using compound 5 (3.54 g, $0.01 \mathrm{~mol})$ as starting material. Yield 78\%.

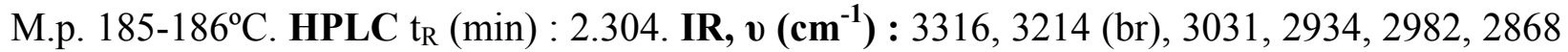
(w), 1701(vs), 1662, 1638 (w), 1615, 1530 (vs), 1431 (w), 1377, 1244 (m). ${ }^{1} \mathbf{H}$ NMR : $\delta$ (ppm) 1.25-1.34 (m, 3H), 2.12-2.36 (m, 5H), 2.65 and $2.71(\mathrm{~s}$ and $\mathrm{s}, 2 \mathrm{H}), 3.85-3.90(\mathrm{q}, 2 \mathrm{H}), 3.91(\mathrm{~s}$, $1 \mathrm{H}), 3.94(\mathrm{~s}, 1 \mathrm{H}), 4.91-4.93$ and 5.43-5.62 (q and $\mathrm{m}, 1 \mathrm{H}), 7.14(\mathrm{~d}, 1 \mathrm{H}, J=7.4 \mathrm{~Hz}), 7.47(\mathrm{t}, 2 \mathrm{H}$, $J=7.2 \mathrm{~Hz}, J=7.8 \mathrm{~Hz}$ ), 7.53-7.58 (q, 1H), 7.85 (t, 2H, $J=7.6 \mathrm{~Hz}, J=8.3 \mathrm{~Hz}$ ), 7.74 and 9.15 (s and $\mathrm{s}, 1 \mathrm{H})$. HR-MS (FAB $\left.{ }^{+}\right), \mathbf{m} / \mathbf{z}: 417.1023\left(\mathrm{M}+\mathrm{Na}, \mathrm{C}_{17} \mathrm{H}_{22} \mathrm{~N}_{4} \mathrm{O}_{3} \mathrm{~S}_{2} \mathrm{Na}\right), 395.1204(\mathrm{M}+\mathrm{H}$, $\left.\mathrm{C}_{17} \mathrm{H}_{23} \mathrm{~N}_{4} \mathrm{O}_{3} \mathrm{~S}_{2}\right), 347.1184\left(\mathrm{C}_{16} \mathrm{H}_{19} \mathrm{~N}_{4} \mathrm{O}_{3} \mathrm{~S}\right), 320.0933\left(\mathrm{C}_{14} \mathrm{H}_{16} \mathrm{~N}_{4} \mathrm{O}_{3} \mathrm{~S}\right)$.

N-\{1-[[2-(3-Allyl-4-oxo-1,3-thiazolidin-2-ylidene)hydrazino]carbonyl]-3-(methylthio)-

propyl \}benzamide (10). Compound 10 was synthesized according to the procedure given above for compounds 8-11 by using compound 6 (3.66 g, $0.01 \mathrm{~mol}$ ) as starting material. Yield 92\%. M.p. $162^{\circ} \mathrm{C}$. HPLC $t_{R}(\mathrm{~min}): 2.480 . \mathbf{I R}, \boldsymbol{v}\left(\mathbf{c m}^{-1}\right): 3304(\mathrm{br}), 3090,3015,2913,2837,2982(\mathrm{w})$, 1723 (s), 1649, 1642 (w), 1618 (vs), 1578 (w), 1532 (vs), 1425, 1381, 1358, 1314 (m), 1269, 1237(m). ${ }^{1} \mathbf{H}$ NMR : $\boldsymbol{\delta}(\mathbf{p p m})$ 1.85-2.13 (m, 5H), 2.41-2.58 (m, 2H), $3.65(\mathrm{~s}, 1 \mathrm{H}), 3.74(\mathrm{~s}, 1 \mathrm{H})$, $4.19(\mathrm{t}, 2 \mathrm{H}, J=5.8 \mathrm{~Hz}, J=9.7 \mathrm{~Hz}), 4.76-4.78$ and 5.26-5.43 (q and $\mathrm{m}, 1 \mathrm{H}), 5.01-5.19(\mathrm{~m}, 2 \mathrm{H})$, 5.63-5.70 (m, 1H), 7.02 and 7.16 (d and d, $1 \mathrm{H}, J=8.0 \mathrm{~Hz}, J=7.7 \mathrm{~Hz}), 7.23-7.27(\mathrm{~m}, 2 \mathrm{H})$, 7.33- 
$7.36(\mathrm{q}, 1 \mathrm{H}), 7.65(\mathrm{t}, 2 \mathrm{H}, J=7.0 \mathrm{~Hz}, J=6.1 \mathrm{~Hz}), 8.00$ and $9.20\left(\mathrm{~s}\right.$ and s, 1H). HR-MS (FAB ${ }^{+}$), $\mathbf{m} / \mathbf{z}: 429.1029 \quad\left(\mathrm{M}+\mathrm{Na}, \mathrm{C}_{18} \mathrm{H}_{22} \mathrm{~N}_{4} \mathrm{O}_{3} \mathrm{~S}{ }_{2} \mathrm{Na}\right), 407.1196\left(\mathrm{M}+\mathrm{H}, \mathrm{C}_{18} \mathrm{H}_{23} \mathrm{~N}_{4} \mathrm{O}_{3} \mathrm{~S}_{2}\right), 332.0945$ $\left(\mathrm{C}_{15} \mathrm{H}_{16} \mathrm{~N}_{4} \mathrm{O}_{3} \mathrm{~S}\right)$.

N-\{1-[[2-(3-Benzyl-4-oxo-1,3-thiazolidin-2-ylidene)hydrazino]carbonyl]-3-(methylthio)propyl\}benzamide (11). Compound 11 was synthesized according to the procedure given above for compounds 8-11 by using compound 7 (4.16 g, $0.01 \mathrm{~mol})$ as starting material. Yield 84\%. M.p. $184^{\circ} \mathrm{C}$. HPLC $t_{R}(\mathrm{~min}): 3.482$. IR, v ( $\left.\mathbf{c m}^{-1}\right)$ : 3246 (br), 3063, 2847, 2985 (w), 1723, 1671, 1632 (s), 1599 (vs), 1534, 1511, 1427, 1383, 1343, 1233 (m). ${ }^{1} \mathbf{H}$ NMR : $\boldsymbol{\delta}$ (ppm) 2.28-2.46 (m, $7 \mathrm{H}), 3.97(\mathrm{~s}, 1 \mathrm{H}), 4.11(\mathrm{~s}, 1 \mathrm{H}), 5.01-5.07(\mathrm{q}, 2 \mathrm{H}), 5.16,5.19$ and 5.62-5.64 (s, s and q, 1H), 7.32 and 7.34 (d and d, $1 \mathrm{H}, J=7.5 \mathrm{~Hz}$ ), 7.41-7.67 (m, 8H), 7.95-7.98 (q, 2H), 7.92 and 9.35 (s and $\mathrm{s}, 1 \mathrm{H})$. HR-MS (FAB $\left.{ }^{+}\right), \mathbf{m} / \mathbf{z}: 479.1209\left(\mathrm{M}+\mathrm{Na}, \mathrm{C}_{22} \mathrm{H}_{24} \mathrm{~N}_{4} \mathrm{O}_{3} \mathrm{~S}_{2} \mathrm{Na}\right), 457.1371(\mathrm{M}+\mathrm{H}$, $\left.\mathrm{C}_{22} \mathrm{H}_{25} \mathrm{~N}_{4} \mathrm{O}_{3} \mathrm{~S}_{2}\right), 409.1320\left(\mathrm{C}_{21} \mathrm{H}_{21} \mathrm{~N}_{4} \mathrm{O}_{3} \mathrm{~S}\right), 382.1089\left(\mathrm{C}_{19} \mathrm{H}_{18} \mathrm{~N}_{4} \mathrm{O}_{3} \mathrm{~S}\right)$.

N-\{1-[[2-[5-(nonsubstituted/substitutedbenzylidene)-3-alkyl/arylalkyl-4-oxo-1,3-thiazolidin2-ylidene]hydrazino]carbonyl]-3-(methylthio)propyl $\}$-benzamides $\quad$ (12-27). N-\{1-[[2-(3alkyl/arylalkyl-4-oxo-1,3-thiazolidin-2-ylidene)hydrazino]carbonyl]-3-(methylthio)propyl -

benzamides (8-11) were heated under reflux for $0.5 \mathrm{~h}$ in the presence of sodium methoxyde ( $0.023 \mathrm{~g} \mathrm{Na} / 10 \mathrm{ml}$ methanol )in methanolic medium. Appropriate aldehydes were added to the reaction medium and again it was heated under reflux for $1 \mathrm{~h}$. Reaction mixture was cooled and poured into ice-cold water and neutralized with glacial acetic acid. The crude products were filtered, dried and washed with boiling ethanol (compounds 12-15, 20-27) or crystallized from ethanol ( compounds 16-19).

N-\{1-[[2-[5-Benzylidene-3-methyl-4-oxo-1,3-thiazolidin-2-ylidene]hydrazino]-carbonyl]-3(methylthio)propyl\}benzamide (12). Synthesis of compound 12 was carrried out by refluxing compound $8(0.380 \mathrm{~g}, 0.001 \mathrm{~mol})$ with benzaldehyde $(99 \%, 0.107 \mathrm{~g}, 0.001 \mathrm{~mol})$ according to the procedure given above for compounds 12-27. Yield 80\%. M.p. 223-225 ${ }^{\circ}$. HPLC $t_{R}(\min )$ : 3.047. IR, v (cm $\left.{ }^{-1}\right)$ : 3252 (br), 3063, 2998, 2913, 2832 (w), 1713 (vs), 1671 (s), 1630,1599 (vs), 1537 (m), 1491 (w), 1427 (m), 1372, 1318 (s), 1219 (w). ${ }^{1}$ H NMR : $\delta$ (ppm) 2.13-2.26 $(\mathrm{m}, 5 \mathrm{H}), 2.61(\mathrm{~d}, 2 \mathrm{H}, J=1.6 \mathrm{~Hz}), 3.41(\mathrm{~s}, 3 \mathrm{H}), 4.87-4.91$ and 5.22-5.61 (q and $\mathrm{m}, 1 \mathrm{H})$, 7.46-7.61 $(\mathrm{m}, 8 \mathrm{H}), 7.74$ and 7.76 (s and s, 1H), 7.95-8.01 (q, 2H), 8.34 and 8.57 (d and d, 1H, $J=7.7 \mathrm{~Hz}$ ), 10.61 and 10.76 (s and s, 1H). HR-MS $\left(\mathbf{E I}^{+}\right), \mathbf{m} / \mathbf{z}: 468.1251\left(\mathrm{M}^{+}, \mathrm{C}_{23} \mathrm{H}_{24} \mathrm{~N}_{4} \mathrm{O}_{3} \mathrm{~S}_{2}\right.$ requires 468.1292), $420.1252 \quad\left(\mathrm{C}_{22} \mathrm{H}_{20} \mathrm{~N}_{4} \mathrm{O}_{3} \mathrm{~S}\right), 394.1093 \quad\left(\mathrm{C}_{20} \mathrm{H}_{18} \mathrm{~N}_{4} \mathrm{O}_{3} \mathrm{~S}\right), 233.0626 \quad\left(\mathrm{C}_{11} \mathrm{H}_{11} \mathrm{~N}_{3} \mathrm{OS}\right)$, $105.0324\left(\mathrm{C}_{5} \mathrm{H}_{3} \mathrm{~N}_{3}\right)$.

N-\{1-[[2-[5-(4-Chlorobenzylidene)-3-methyl-4-oxo-1,3-thiazolidin-2-ylidene]-hydrazino]carbonyl]-3-(methylthio)propyl benzamide (13). Synthesis of compound 13 was carrried out by refluxing compound $8(0.380 \mathrm{~g}, 0.001 \mathrm{~mol})$ with 4-chlorobenzaldehyde $(0.140 \mathrm{~g}, 0.001 \mathrm{~mol})$ according to the procedure given above for compounds 12-27. Yield 83\%. M.p. $250-254^{\circ} \mathrm{C}$. HPLC $\mathrm{t}_{\mathrm{R}}(\mathrm{min}): 6.958,7.529,8.162 . \mathbf{I R}, \boldsymbol{v}\left(\mathbf{c m}^{-1}\right): 3247(\mathrm{br}), 3069,3001,2916,2832(\mathrm{w})$, 1717, 1672 (m) 1630, 1599 (s), 1539 (m), 1493 (w), 1427 (m), 1373 (s), 1318 (m), 1217 (w), 1098, 819 (m). ${ }^{1} \mathbf{H}$ NMR : $\boldsymbol{\delta}(\mathbf{p p m})$ 2.07-2.24 (m, 5H), $2.56(\mathrm{~s}, 2 \mathrm{H}), 3.36(\mathrm{~s}, 3 \mathrm{H}), 4.86-4.87$ and 5.31-5.49 (q and $\mathrm{m}, 1 \mathrm{H}), 7.41-7.53$ and $7.66(\mathrm{~m}$ and $\mathrm{s}, 7 \mathrm{H}), 7.75(\mathrm{~s}, 1 \mathrm{H}), 7.91-7.93(\mathrm{q}, 2 \mathrm{H})$, 
$7.90,8.04$ and 8.38 (d, d, and d, $J=7.85 \mathrm{~Hz}$ ), 10.48 and 10.66 (s and s, 1H). ${ }^{13}$ C NMR-DEPT : $\boldsymbol{\delta}$ (ppm) $15.2\left(\mathrm{CH}_{3}\right), 29.9,30.1\left(\mathrm{CH}_{3}\right), 30.5\left(\mathrm{CH}_{2}\right), 31.6\left(\mathrm{CH}_{2}\right), 52.4(\mathrm{CH}), 127.9(\mathrm{CH}), 128.1$ $(\mathrm{CH}), 128.6(\mathrm{CH}), 128.7(\mathrm{CH}), 129.7(\mathrm{CH}), 131.7(\mathrm{CH}), 131.9(\mathrm{CH}), 132.7\left(\mathrm{C}_{\mathrm{q}}\right), 134.4\left(\mathrm{C}_{\mathrm{q}}\right)$, $135.1\left(\mathrm{C}_{\mathrm{q}}\right), 152.6\left(\mathrm{C}_{\mathrm{q}}\right), 165.5\left(\mathrm{C}_{\mathrm{q}}\right), 167.2\left(\mathrm{C}_{\mathrm{q}}\right), 168.8\left(\mathrm{C}_{\mathrm{q}}\right)$. HR-MS $\left(\mathbf{E I}^{+}\right), \mathbf{m} / \mathbf{z}: 504.0892(\mathrm{M}+2$, $\left.\mathrm{C}_{23} \mathrm{H}_{23}{ }^{37} \mathrm{ClN}_{4} \mathrm{O}_{3} \mathrm{~S}_{2}\right), \quad 502.0892 \quad\left(\mathrm{M}^{+}, \quad \mathrm{C}_{23} \mathrm{H}_{23}{ }^{35} \mathrm{ClN}_{4} \mathrm{O}_{3} \mathrm{~S}_{2}\right.$ requires 508.0900), 430.0709 $\left(\mathrm{C}_{20} \mathrm{H}_{17}{ }^{37} \mathrm{ClN}_{4} \mathrm{O}_{3} \mathrm{~S}\right), 428.0736\left(\mathrm{C}_{20} \mathrm{H}_{17}{ }^{35} \mathrm{ClN}_{4} \mathrm{O}_{3} \mathrm{~S}\right), 267.0213\left(\mathrm{C}_{11} \mathrm{H}_{10}{ }^{35} \mathrm{ClN}_{3} \mathrm{OS}\right)$.

N-\{1-[[2-[5-(2-Fluorobenzylidene)-3-methyl-4-oxo-1,3-thiazolidin-2-ylidene]-hydrazino]carbonyl]-3-(methylthio)propyl \}benzamide (14). Synthesis of compound 14 was carrried out by refluxing compound $8(0.380 \mathrm{~g}, 0.001 \mathrm{~mol})$ with 2-fluorobenzaldehyde $(98 \%, 0.126 \mathrm{~g}, 0.001$ mol) according to the procedure given above for compounds 12-27. Yield 88\%. M.p. 244-246 ${ }^{\circ} \mathrm{C}$. HPLC $t_{R}(\min ): 5.170,5.768$. IR, v $\left(\mathbf{c m}^{-1}\right): 3254(\mathrm{br}), 3063,2997,2919,2832$ (w), 1714, 1667, 1630, 1598, 1578 (s), 1483, 1452, 1426, 1370, 1318, 1239 (m), 1216, 1189, 1131 (w), 931, 848 (w). ${ }^{1} \mathbf{H}$ NMR : $\boldsymbol{\delta}$ (ppm) 2.24 and 2.31 (s,1H and s, 2H), 2.34-2.49 (m, 2H), 2.76-2.85 and 2.93-2.97 ( $\mathrm{m}$ and $\mathrm{m}, 2 \mathrm{H}$ ), 3.54 and 3.57 (s and s, 3H), 5.03-5.08 and 5.62-5.82 ( $\mathrm{q}$ and $\mathrm{m}$, 1H), 7.24-7.31, 7.36-7.45, 7.54-7.61 and 7.63-7.69 (q ,2s, m ad m, 8H), 7.95 (d, 2H, J =7.4 Hz), 8.12 and 8.16 ( $\mathrm{s}$ and $\mathrm{s}, 1 \mathrm{H}$ ), 7.91 and 9.49 ( $\mathrm{s}$ and $\mathrm{s}, 1 \mathrm{H})$. HR-MS $\left(\mathbf{E I}^{+}\right), \mathbf{m} / \mathbf{z}: 486.1208\left(\mathrm{M}^{+}\right.$, $\mathrm{C}_{23} \mathrm{H}_{23} \mathrm{~F} \quad \mathrm{~N}_{4} \mathrm{O}_{3} \mathrm{~S}_{2}$ requires 486.1214), $412.1012\left(\mathrm{C}_{20} \mathrm{H}_{17} \mathrm{FN}_{4} \mathrm{O}_{3} \mathrm{~S}\right), 251.0544\left(\mathrm{C}_{11} \mathrm{H}_{10} \mathrm{FN}_{3} \mathrm{OS}\right)$, $105.0344\left(\mathrm{C}_{7} \mathrm{H}_{5} \mathrm{O}\right)$.

N-\{1-[[2-[5-(4-Fluorobenzylidene)-3-methyl-4-oxo-1,3-thiazolidin-2-ylidene]- hydrazino]carbonyl]-3-(methylthio)propyl benzamide (15). Synthesis of compound 15 was carrried out by refluxing compound $8(0.380 \mathrm{~g}, 0.001 \mathrm{~mol})$ with 4-fluorobenzaldehyde $(0.124 \mathrm{~g}, 0.001 \mathrm{~mol})$ according to the procedure given above for compounds 12-27. Yield 56\%. M.p. $240-241^{\circ} \mathrm{C}$. HPLC $t_{R}(\mathrm{~min}):$ 7.965. IR, v (cm $\left.{ }^{-1}\right)$ : 3251 (br), 3063, 3001, 2928, 2826 (w), 1712, 1667 (s), 1631, 1602 (vs), 1509 (s), 1425 (m), 1370 (vs), 1319, 1238 (s), 1162 (m), 1124, 826 (s). ${ }^{1} \mathbf{H}$ NMR : $\boldsymbol{\delta}$ (ppm) 2.14 and 2.22 (s, $1 \mathrm{H}$ and s, 2H), 2.26-2.38 (m, 2H), 2.67-2.76 and 2.81-2.98 ( $\mathrm{m}$ and $\mathrm{m}, 2 \mathrm{H}), 3.44$ and 3.51 ( $\mathrm{s}$ and $\mathrm{s}, 3 \mathrm{H}), 4.92-4.97$ and 5.49-5.71 ( $\mathrm{q}$ and $\mathrm{m}, 1 \mathrm{H}), 7.12(\mathrm{~s}, 1 \mathrm{H})$, 7.16-7.23 (q, 2H), 7.47-7.61 (m, 5H ), 7.72-7.76 and $7.82(\mathrm{~s}$ and $\mathrm{s}, 1 \mathrm{H}), 7.86(\mathrm{~d}, 2 \mathrm{H}, J=7.5$ $\mathrm{Hz}$ ), 7.72 and 9.29 ( $\mathrm{s}$ and $\mathrm{s}, 1 \mathrm{H})$. HR-MS $\left(\mathbf{F A B}^{+}\right), \mathbf{m} / \mathbf{z}: 509.1084\left(\mathrm{M}+\mathrm{Na}, \mathrm{C}_{23} \mathrm{H}_{23} \mathrm{FN}_{4} \mathrm{O}_{3} \mathrm{~S}_{2} \mathrm{Na}\right)$, $487.1216\left(\mathrm{M}+\mathrm{H}, \mathrm{C}_{23} \mathrm{H}_{24} \mathrm{FN}_{4} \mathrm{O}_{3} \mathrm{~S}_{2}\right)$.

N-\{1-[[2-[5-Benzylidene-3-ethyl-4-oxo-1,3-thiazolidin-2-ylidene]hydrazino]-carbonyl]-3(methylthio)propyl\}benzamide (16). Synthesis of compound 16 was carrried out by refluxing compound 9 (0.394 g, $0.001 \mathrm{~mol})$ with benzaldehyde $(99 \%, 0.107 \mathrm{~g}, 0.001 \mathrm{~mol})$ according to the procedure given above for compounds 12-27. Yield 80\%. M.p. 222-224 ${ }^{\circ} \mathrm{C}$. HPLC $\mathrm{t}_{\mathrm{R}}(\mathrm{min})$ : 3.523, 5.050. IR, v (cm $\left.{ }^{-1}\right)$ : 3269, 3166 (br), 3025, 2977, 2917 (w), 1711 (vs), 1682 (m), 1636 1595 (vs), 1535 (s), 1437, 1373, 1341 (m), 1242 (s). ${ }^{1} \mathbf{H}$ NMR : $\boldsymbol{\delta}$ (ppm) 1.28 and 1.38 (t and t, $3 \mathrm{H}, J=7.1 \mathrm{~Hz}, J=7.1 \mathrm{~Hz}$ and $J=7.1 \mathrm{~Hz}, J=7.1 \mathrm{~Hz}), 2.06-2.41(\mathrm{~m}, 5 \mathrm{H}), 2.67$ and 2.73 (s and s, $2 \mathrm{H}), 3.94-4.04(\mathrm{~m}, 2 \mathrm{H}), 5.07-5.11$ and 5.48-5.68 $(\mathrm{q}$ and $\mathrm{m}, 1 \mathrm{H}), 7.24$ and 7.40-7.54 (d, J=7.92 $\mathrm{Hz}$ and $\mathrm{m}, 9 \mathrm{H}$ ), 7.73 and 7.81 (s and s, 1H), 7.85 (d, 2H, J=7.3 Hz), 8.39 and 9.69 (s and s, 1H). HR-MS $\left(\mathbf{E I}^{+}\right), \mathbf{m} / \mathbf{z}: 482.1427\left(\mathrm{M}^{+}, \mathrm{C}_{24} \mathrm{H}_{26} \mathrm{~N}_{4} \mathrm{O}_{3} \mathrm{~S}_{2}\right.$ requires 482.1448), 408.1251 $\left(\mathrm{C}_{21} \mathrm{H}_{20} \mathrm{~N}_{4} \mathrm{O}_{3} \mathrm{~S}\right), 247.0776\left(\mathrm{C}_{12} \mathrm{H}_{13} \mathrm{~N}_{3} \mathrm{OS}\right), 105.0330\left(\mathrm{C}_{7} \mathrm{H}_{5} \mathrm{O}\right)$. 
N-\{1-[[2-[5-(4-Chlorobenzylidene)-3-ethyl-4-oxo-1,3-thiazolidin-2-ylidene]-hydrazino]carbonyl]-3-(methylthio)propyl \}benzamide (17). Synthesis of compound 17 was carrried out by refluxing compound $9(0.394 \mathrm{~g}, 0.001 \mathrm{~mol})$ with 4-chlorobenzaldehyde $(0.140 \mathrm{~g}, 0.001 \mathrm{~mol})$ according to the procedure given above for compounds 12-27. Yield 29\%. M.p. $200^{\circ} \mathrm{C}$. HPLC $\mathrm{t}_{\mathrm{R}}(\mathrm{min}): 10.120 . \mathbf{I R}, \mathbf{v}\left(\mathbf{c m}^{-1}\right)$ : 3305 (br), 3029, 2973,2921 (w), $1701(\mathrm{vs}), 1655(\mathrm{w}), 1630$, 1532 (vs), 1487, 1437 (m), 1397, 1345, 1242 (s), 1096 (m), 816 (w). ${ }^{1}$ H NMR : $\delta$ (ppm) 1.42$1.50(\mathrm{~m}, 3 \mathrm{H}), 2.22-2.49(\mathrm{~m}, 5 \mathrm{H}), 2.69-2.89$ and 2.89-3.09 (m and $\mathrm{m}, 2 \mathrm{H}), 4.01-4.25(\mathrm{q}, 2 \mathrm{H})$, 4.98-5.21 and 5.59-5.79 (q and s,1H), 7.30, (d, $J=7.5 \mathrm{~Hz}, 1 \mathrm{H}), 7.54-7.61$ and 7.62-7.70 (m and $\mathrm{m}, 7 \mathrm{H}$ ), 7.81 and 7.96 (s and $\mathrm{t}, J=1.3 \mathrm{~Hz}, J=7.3 \mathrm{~Hz}, 3 \mathrm{H}$ ), 7.88 and 9.49 (s and s, 1H). HR-MS $\left(\mathbf{F A B}^{+}\right), \quad \mathbf{m} / \mathbf{z} \quad: \quad 541.0919 \quad\left(\mathrm{M}+\mathrm{Na}, \quad \mathrm{C}_{24} \mathrm{H}_{25}{ }^{37} \mathrm{ClN}_{4} \mathrm{O}_{3} \mathrm{~S}_{2} \mathrm{Na}\right), \quad 539.0900 \quad(\mathrm{M}+\mathrm{Na}$, $\left.\mathrm{C}_{24} \mathrm{H}_{25}{ }^{35} \mathrm{ClN}_{4} \mathrm{O}_{3} \mathrm{~S}_{2} \mathrm{Na}\right), 519.1092\left(\mathrm{M}+\mathrm{H}, \mathrm{C}_{24} \mathrm{H}_{26}{ }^{37} \mathrm{ClN}_{4} \mathrm{O}_{3} \mathrm{~S}_{2}\right), 518.1056(\mathrm{M}+2), 517.1114(\mathrm{M}+\mathrm{H}$, $\left.\mathrm{C}_{24} \mathrm{H}_{26}{ }^{35} \mathrm{ClN}_{4} \mathrm{O}_{3} \mathrm{~S}_{2}\right)$.

\section{N-\{1-[[2-[5-(2-Fluorobenzylidene)-3-ethyl-4-oxo-1,3-thiazolidin-2-ylidene]-hydrazino]-} carbonyl]-3-(methylthio)propyl benzamide (18). Synthesis of compound $\mathbf{1 8}$ was carrried out by refluxing compound $9(0.394 \mathrm{~g}, 0.001 \mathrm{~mol})$ with 2-fluorobenzaldehyde $(98 \%, 0.126 \mathrm{~g}, 0.001$ mol) according to the procedure given above for compounds 12-27. Yield 74\%. M.p. $220-222^{\circ} \mathrm{C}$.

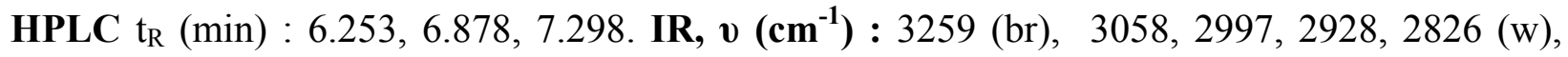
1717 (s), 1669 (m), 1630, 1599 (vs), 1537 (s), 1480, 1447 (m), 1377 (s), 1312 (w), 1240 (s), $1190(\mathrm{w}), 1140(\mathrm{~s}), 932(\mathrm{w}) .{ }^{1} \mathbf{H}$ NMR : $\boldsymbol{\delta}(\mathbf{p p m}) 1.28$ and $1.38(\mathrm{t}$ and t, 3H, $J=7.1 \mathrm{~Hz}, \mathrm{~J}=7.1$ and $J=7.0 H z, J=7.0 H z), 2.11$ and $2.16(\mathrm{~s}, 1 \mathrm{H}$ and $\mathrm{s}, 2 \mathrm{H}), 2.24-2.39(\mathrm{~m}, 2 \mathrm{H}), 2.65-2.79(\mathrm{~m}$, $2 \mathrm{H}), 3.94-4.06(\mathrm{~m}, 2 \mathrm{H})$, 5.07-5.13 and 5.47-5.68 (q and $\mathrm{m}, 1 \mathrm{H}), 7.12-7.26(\mathrm{~m}, 3 \mathrm{H}), 7.36-7.53$ $(\mathrm{m}, 5 \mathrm{H}), 7.83(\mathrm{~d}, 2 \mathrm{H}, J=7.6 \mathrm{~Hz}), 7.93$ and 8.01 (s and s, 1H), 8.55 and 9.85 (s and s, 1H). HRMS (EI $\left.{ }^{+}\right), \mathbf{m} / \mathbf{z}: 500.1326\left(\mathrm{M}^{+}, \mathrm{C}_{24} \mathrm{H}_{25} \mathrm{FN}_{4} \mathrm{O}_{3} \mathrm{~S}_{2}\right.$ requires 500.1371), $426.1161\left(\mathrm{C}_{21} \mathrm{H}_{19} \mathrm{FN}_{4} \mathrm{O}_{3} \mathrm{~S}\right)$, $265.0683\left(\mathrm{C}_{12} \mathrm{H}_{12} \mathrm{FN}_{3} \mathrm{OS}\right), 105.0349\left(\mathrm{C}_{7} \mathrm{H}_{5} \mathrm{O}\right)$.

N-\{1-[[2-[5-(4-Fluorobenzylidene)-3-ethyl-4-oxo-1,3-thiazolidin-2-ylidene]hydrazino]carbonyl]-3-(methylthio)propyl benzamide (19). Synthesis of compound 19 was carrried out by refluxing compound $9(0.394 \mathrm{~g}, 0.001 \mathrm{~mol})$ with 4-fluorobenzaldehyde $(0.124 \mathrm{~g}, 0.001 \mathrm{~mol})$ according to the procedure given above for compounds 12-27. Yield 43\%. M.p. 200-202 ${ }^{\circ}$.

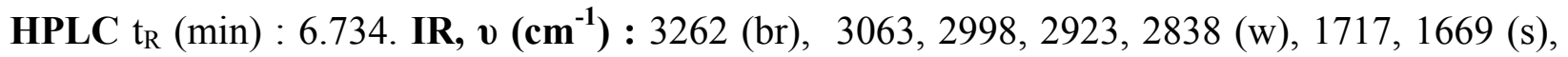
1632, 1601 (vs), 1537, 1237 (s), 1161, 1128, 826 (C-H, m). ${ }^{\mathbf{H}} \mathbf{N M R}: \boldsymbol{\delta}$ (ppm) 1.31 and 1.38 (t, $J=7,0 \mathrm{~Hz}, J=7,0 \mathrm{~Hz}$ and $\mathrm{t}, J=7.0 \mathrm{~Hz}, J=6.7 \mathrm{~Hz}, 3 \mathrm{H}$ ), 2.12 and 2.20 (s and s, 3H), 2.25-2.42 (m, $2 \mathrm{H}), 2.67,2.74$ and $2.83(\mathrm{~s}, \mathrm{~s}$ and $\mathrm{s}, 2 \mathrm{H}), 3.82-4.05(\mathrm{~m}, 2 \mathrm{H}), 4.99-5.05$ and 5.43-5.71 (q and s, $1 \mathrm{H}), 7.15,7.25$ and 7.44-7.57 (t, $J=8.5 \mathrm{~Hz}, J=8.5 \mathrm{~Hz}, \mathrm{~d}, J=5.8 \mathrm{~Hz}$ and $\mathrm{m}, 8 \mathrm{H}$ ), 7.71 and 7.79 (s and s, 1H), 7.85 (d, 2H, J=7.3 Hz), $8.01,9.04,9.47(\mathrm{~s}, \mathrm{~s}, \mathrm{~s}, 1 \mathrm{H})$. HR-MS (EI $\left.{ }^{+}\right), \mathbf{~ m} / \mathbf{z}: 500.1314$ $\left(\mathrm{M}^{+}, \mathrm{C}_{24} \mathrm{H}_{25} \mathrm{FN}_{4} \mathrm{O}_{3} \mathrm{~S}_{2}\right.$ requires 500.1371), $426.1152 \quad\left(\mathrm{C}_{21} \mathrm{H}_{19} \mathrm{~N}_{4} \mathrm{FO}_{3} \mathrm{~S}\right), 265.0715\left(\mathrm{C}_{12} \mathrm{H}_{12} \mathrm{FN}_{3} \mathrm{OS}\right)$, $105.0321\left(\mathrm{C}_{7} \mathrm{H}_{5} \mathrm{O}\right)$.

N-\{1-[[2-[5-Benzylidene-3-allyl-4-oxo-1,3-thiazolidin-2-ylidene]hydrazino]-carbonyl]-3(methylthio)propyl\}benzamide (20). Synthesis of compound 20 was carried out by refluxing compound 10 (0.406 g, $0.001 \mathrm{~mol})$ with benzaldehyde $(99 \%, 0.107 \mathrm{~g}, 0.001 \mathrm{~mol})$ according to the procedure given above for compounds 12-27. Yield 35\%. M.p. 203-205 ${ }^{\circ}$ C. HPLC $t_{R}(\min )$ : 
7.754. IR, v (cm $\left.{ }^{-1}\right)$ : 3266, 3175 (br), 3027, 2975, 2915 (w), 1717 (s), $1678(\mathrm{~m}), 1632,1593$ (vs), 1534 (s), 1491 (w), 1426 (m), 1379 (s), 1356, 1331, 1223 (w). ${ }^{1}$ H NMR : $\delta$ (ppm) 1.91$2.19(\mathrm{~m}, 5 \mathrm{H}), 2.42-2.65(\mathrm{~m}, 2 \mathrm{H}), 4.32-4.39(\mathrm{~m}, 2 \mathrm{H}), 4.75-4.91$ and 5.31-5.45 (q and $\mathrm{m}, 1 \mathrm{H})$, 5.02-5.23 (m, 2H), 5.68-5.75 (m, 1H), 7.02 and $7.11(\mathrm{~d}, J=8.0 \mathrm{~Hz}$ and $\mathrm{d}, J=7.7 \mathrm{~Hz}, 1 \mathrm{H}), 7.22-$ 7.36 and $7.56(\mathrm{~m}$ and s, 9H), $7.65(\mathrm{~d}, J=6.9 \mathrm{~Hz}, 2 \mathrm{H}), 8.00$ and 9.35 ( $\mathrm{s}$ and $\mathrm{s}, 1 \mathrm{H})$. HR-MS (EI ${ }^{+}$), $\mathbf{m} / \mathbf{z}$ : $494.1457\left(\mathrm{M}^{+}, \mathrm{C}_{25} \mathrm{H}_{26} \mathrm{~N}_{4} \mathrm{O}_{3} \mathrm{~S}_{2}\right.$ requires 494.1448), $420.1290\left(\mathrm{C}_{22} \mathrm{H}_{20} \mathrm{~N}_{4} \mathrm{O}_{3} \mathrm{~S}\right), 259.0782$ $\left(\mathrm{C}_{13} \mathrm{H}_{13} \mathrm{~N}_{3} \mathrm{OS}\right), 105.0339\left(\mathrm{C}_{7} \mathrm{H}_{5} \mathrm{O}\right)$.

N-\{1-[[2-[5-(4-Chlorobenzylidene)-3-allyl-4-oxo-1,3-thiazolidin-2-ylidene]-hydrazino]carbonyl]-3-(methylthio)propyl $\}$ benzamide (21). Synthesis of compound 21 was carrried out by refluxing compound $10(0.406 \mathrm{~g}, 0.001 \mathrm{~mol})$ with 4-chlorobenzaldehyde $(0.140 \mathrm{~g}, 0.001 \mathrm{~mol})$ according to the procedure given above for compounds 12-27. Yield 35\%. M.p. 220-222 ${ }^{\circ} \mathrm{C}$.

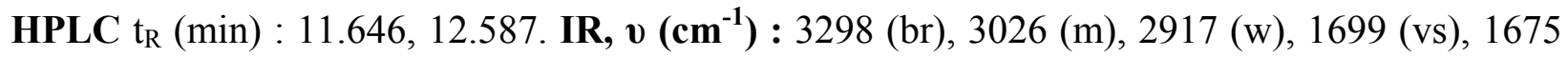
(w), 1628, 1526 (vs), 1489 (s), 1427 (m), 1385 (s), 1360, 1231 (w), 1086, 822 (m). ${ }^{1} \mathbf{H}$ NMR : $\boldsymbol{\delta}$ (ppm) 2.01 and $2.06(\mathrm{~s}, 1 \mathrm{H}$ and s, 2H), 2.11-2.15 (m, 2H), 2.52-2.73 (m, 2H), $4.44(\mathrm{~d}, \mathrm{~J}=5.6 \mathrm{~Hz}$, $2 \mathrm{H}), 4.69-4.86$ and 5.24-5.32 (q and s, $1 \mathrm{H}), 5.13-5.23(\mathrm{~m}, 2 \mathrm{H}), 5.73-5.97(\mathrm{~m}, 1 \mathrm{H}), 7.36-7.47(\mathrm{~m}$, $7 \mathrm{H}), 7.61$ and $7.68(\mathrm{~s}$ and $\mathrm{s}, 1 \mathrm{H}), 7.80-7.92(\mathrm{q}, 2 \mathrm{H}), 8.03$ and 8.33 (d and d, J=7.8 Hz, 1H), 10.45 and 10.60 ( $\mathrm{s}$ and $\mathrm{s}, 1 \mathrm{H})$. HR-MS (EI $\left.{ }^{+}\right), \mathbf{m} / \mathbf{z}: 530.1043\left(\mathrm{M}+2, \mathrm{C}_{25} \mathrm{H}_{25}{ }^{37} \mathrm{ClN}_{4} \mathrm{O}_{3} \mathrm{~S}_{2}\right)$, $528.1055\left(\mathrm{M}^{+}, \mathrm{C}_{25} \mathrm{H}_{25}{ }^{35} \mathrm{ClN}_{4} \mathrm{O}_{3} \mathrm{~S}_{2}\right.$ requires 528.1056), $456.0844\left(\mathrm{C}_{22} \mathrm{H}_{19}{ }^{37} \mathrm{ClN}_{4} \mathrm{O}_{3} \mathrm{~S}\right), 454.0871$ $\left(\mathrm{C}_{22} \mathrm{H}_{19}{ }^{35} \mathrm{ClN}_{4} \mathrm{O}_{3} \mathrm{~S}\right), 295.0373\left(\mathrm{C}_{13} \mathrm{H}_{12}{ }^{37} \mathrm{ClN}_{3} \mathrm{OS}\right), 293.0389\left(\mathrm{C}_{13} \mathrm{H}_{12}{ }^{35} \mathrm{ClN}_{3} \mathrm{OS}\right)$.

\section{N-\{1-[[2-[5-(2-Fluorobenzylidene)-3-allyl-4-oxo-1,3-thiazolidin-2-ylidene]hydrazino]-} carbonyl]-3-(methylthio)propyl $\}$ benzamide (22). Synthesis of compound 22 was carrried out by refluxing compound $10(0.406 \mathrm{~g}, 0.001 \mathrm{~mol})$ with 2-fluorobenzaldehyde $(98 \%, 0.126 \mathrm{~g}, 0.001$ mol) according to the procedure given above for compounds 12-27.Yield 98\%. M.p. $218-220^{\circ} \mathrm{C}$. HPLC $t_{R}$ (min) : 7.649, 8.116. IR, v (cm $\left.{ }^{-1}\right): 3246$ (br), 3021, 2915, 2834 (w), 1709 (s), 1682 (w), 1636, 1600 (vs), 1560 (w), 1509 (vs), 1490, 1458 (w), 1425 (m), 1380, 1238 (s), 1162 , 1136, 926, 832 (m). ${ }^{1} \mathbf{H}$ NMR : $\boldsymbol{\delta}$ (ppm) 2.11 and 2.19 (s and s, 3H), 2.26-2.39 (m, 2H), 2.64$2.83(\mathrm{~m}, 2 \mathrm{H}), 4.52-4.59(\mathrm{~m}, 2 \mathrm{H}), 4.95-5.12$ and 5.48-5.64 (q and $\mathrm{m}, 1 \mathrm{H}), 5.22-5.43(\mathrm{~m}, 2 \mathrm{H})$, 5.88-5.95 $(\mathrm{m}, 1 \mathrm{H}), 7.14-7.21,7.31$ and 7.43-7.57 (m, s and $\mathrm{m}, 8 \mathrm{H}), 7.72$ and $7.81(\mathrm{~s}$ and $\mathrm{s}, 1 \mathrm{H})$, 7.82-7.92 (q, 2H), 8.14 and 9.54 ( $\mathrm{s}$ and $\mathrm{s}, 1 \mathrm{H})$. HR-MS (EI'), m/z : $512.1379\left(\mathrm{M}^{+}\right.$, $\mathrm{C}_{25} \mathrm{H}_{25} \mathrm{FN}_{4} \mathrm{O}_{3} \mathrm{~S}_{2}$ requires 512.1371), $438.1180\left(\mathrm{C}_{22} \mathrm{H}_{19} \mathrm{FN}_{4} \mathrm{O}_{3} \mathrm{~S}\right), 277.0691 \quad\left(\mathrm{C}_{13} \mathrm{H}_{12} \mathrm{FN}_{3} \mathrm{OS}\right)$, $105.0323\left(\mathrm{C}_{5} \mathrm{H}_{3} \mathrm{~N}_{3}\right)$.

N-\{1-[[2-[5-(4-Fluorobenzylidene)-3-allyl-4-oxo-1,3-thiazolidin-2-ylidene]hydrazino]carbonyl]-3-(methylthio)propyl $\}$ benzamide (23). Synthesis of compound 23 was carrried out by refluxing compound $10(0.406 \mathrm{~g}, 0.001 \mathrm{~mol})$ with 4-fluorobenzaldehyde $(0.124 \mathrm{~g}, 0.001 \mathrm{~mol})$ according to the procedure given above for compounds 12-27. Yield 42\%.M.p. 215-216 ${ }^{\circ}$. HPLC $t_{R}(\min ): 7.716,8.186 . \mathbf{I R}, \boldsymbol{v}\left(\mathbf{c m}^{-1}\right): 3250(\mathrm{br}), 3059,3021,2913,2830(\mathrm{w}), 1710(\mathrm{~s})$, 1663 (w), 1633, 1600 (s), 1533 (w), 1510 (s), 1425 (w), 1381 (s), 1350 (w), 1239 (s), 1162 (m), $1136(\mathrm{w}), 833(\mathrm{~s}) .{ }^{\mathbf{1}} \mathbf{H}$ NMR : $\boldsymbol{\delta}$ (ppm) 2.11 and 2.18 (s and s, 3H), 2.22-2.47 (m, 2H), 2.64-2.81 $(\mathrm{q}, 2 \mathrm{H}), 4.51-4.63(\mathrm{~m}, 2 \mathrm{H})$, 5.03-5.08 and 5.53-5.58 (q and $\mathrm{m}, 1 \mathrm{H}), 5.21-5.43(\mathrm{~m}, 2 \mathrm{H}), 5.85-$ $5.98(\mathrm{~m}, 1 \mathrm{H}), 7.00$ and $7.36(\mathrm{~d}$ and $\mathrm{d}, J=7.8 \mathrm{~Hz}, 1 \mathrm{H}), 7.13-7.23(\mathrm{~m}, 2 \mathrm{H}), 7.42-7.62(\mathrm{~m}, 5 \mathrm{H}), 7.71$ 
and 7.79 (s and s, 1H), $7.85(\mathrm{~d}, J=8.5 \mathrm{~Hz}, 2 \mathrm{H}), 8.32,9.66$ and 9.91 (s, s and s, 1H). HR-MS $\left(\mathbf{E I}^{+}\right), \mathbf{m} / \mathbf{z}: 512.1360\left(\mathrm{M}^{+}, \mathrm{C}_{25} \mathrm{H}_{25} \mathrm{FN}_{4} \mathrm{O}_{3} \mathrm{~S}_{2}\right.$ requires 512.1371), $438.1151\left(\mathrm{C}_{22} \mathrm{H}_{19} \mathrm{FN}_{4} \mathrm{O}_{3} \mathrm{~S}\right)$, $277.0680\left(\mathrm{C}_{13} \mathrm{H}_{12} \mathrm{FN}_{3} \mathrm{OS}\right), 105.0327\left(\mathrm{C}_{5} \mathrm{H}_{3} \mathrm{~N}_{3}\right)$.

N-\{1-[[2-[5-Benzylidene-3-benzyl-4-oxo-1,3-thiazolidin-2-ylidene]-hydrazino]carbonyl]-3(methylthio)propyl\}benzamide (24). Synthesis of compound 24 was carried out by refluxing compound 11 (0.456 g, $0.001 \mathrm{~mol})$ with benzaldehyde $(99 \%, 0.107 \mathrm{~g}, 0.001 \mathrm{~mol})$ according to the procedure given above for compounds 12-27. Yield 48\%. M.p. 205-207 ${ }^{\circ}$. HPLC $t_{R}(\min )$ : 29.899. IR, v (cm $\left.{ }^{-1}\right)$ : 3233 (br), 3061, 3036, 2982, 2921, 2838 (w), 1714 (s), 1665 (m), 1638, 1603 (s), 1512, 1431, 1387, 1339 (m), 1312 (w), 1238 (m). ${ }^{1} \mathbf{H}$ NMR : $\boldsymbol{\delta}$ (ppm) 2.04, 2.12 and $2.21(\mathrm{~s}, \mathrm{~s}$ and $\mathrm{s}, 3 \mathrm{H}), 2.24-2.37(\mathrm{~m}, 2 \mathrm{H}), 2.42-2.61,2.62-2.78$ and 2.79-2.94 (m, $\mathrm{m}$ and $\mathrm{m}, 2 \mathrm{H})$, 4.92-5.02 and 5.49-5.59 (q and $\mathrm{m}, 1 \mathrm{H}), 5.04-5.24(5 \mathrm{~s}, 2 \mathrm{H}), 7.14-7.19(\mathrm{q}, 1 \mathrm{H}), 7.31-7.58(\mathrm{~m}$, 13H), 7.79 and 7.86 (s and $\mathrm{t}, J=1.4 \mathrm{~Hz}, J=7.2 \mathrm{~Hz}, 3 \mathrm{H}$ ) 7.91 and 9.35 (s and s, 1H). HR-MS (EI $\left.{ }^{+}\right), \mathbf{m} / \mathbf{z}: 544.1600\left(\mathrm{M}^{+}, \mathrm{C}_{29} \mathrm{H}_{28} \mathrm{~N}_{4} \mathrm{O}_{3} \mathrm{~S}_{2}\right.$ requires 544.1605), $470.1318\left(\mathrm{C}_{26} \mathrm{H}_{22} \mathrm{~N}_{4} \mathrm{O}_{3} \mathrm{~S}\right)$, 309.0954 $\left(\mathrm{C}_{17} \mathrm{H}_{15} \mathrm{~N}_{3} \mathrm{OS}\right), 105.0336\left(\mathrm{C}_{7} \mathrm{H}_{5} \mathrm{O}\right)$.

N-\{1-[[2-[5-(4-Chlorobenzylidene)-3-benzyl-4-oxo-1,3-thiazolidin-2-ylidene]hydrazino]carbonyl]-3-(methylthio)propyl \}benzamide (25). Synthesis of compound 25 was carried out by refluxing compound 11 (0.456 g, $0.001 \mathrm{~mol})$ with 4-chlorobenzaldehyde $(0.140 \mathrm{~g}, 0.001 \mathrm{~mol})$ according to the procedure given above for compounds 12-27. Yield 83\%. M.p. 247-249 ${ }^{\circ} \mathrm{C}$.

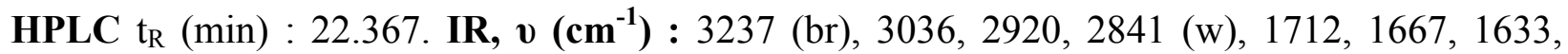
1602, 1490 (s), 1430 (m), 1387, 1340 (s), 1313 (m), 1282 (w), 1084, 823 (C-H, s). ${ }^{1}$ H NMR : $\boldsymbol{\delta}$ (ppm) 1.85-2.38 (m, 5H), 2.43-2.62, 2.63-2.79 and 2.79-2.95 (m, $\mathrm{m}$ and $\mathrm{m}, 2 \mathrm{H})$, 4.90-5.02 and 5.46-5.61 (q and $\mathrm{m}, 1 \mathrm{H}), 5.02-5.27(6 \mathrm{~s}, 2 \mathrm{H}), 7.14-7.18(\mathrm{q}, 1 \mathrm{H}), 7.32-7.65$ and $7.73(\mathrm{~m}$ and $\mathrm{s}$, 13H), $7.86(\mathrm{~d}, 2 \mathrm{H}, J=7.7 \mathrm{~Hz}), 7.76,7.77$ and 9.37 (s ,s and s, 1H). HR-MS (EI $\left.{ }^{+}\right), \mathbf{m} / \mathbf{z}: 580.1193$ $\left(\mathrm{M}+2, \mathrm{C}_{29} \mathrm{H}_{27}{ }^{37} \mathrm{ClN}_{4} \mathrm{O}_{3} \mathrm{~S}_{2}\right), 578.1201\left(\mathrm{M}^{+}, \mathrm{C}_{29} \mathrm{H}_{27}{ }^{35} \mathrm{ClN}_{4} \mathrm{O}_{3} \mathrm{~S}_{2}\right.$ requires 578.1213), 532.1130 $\left(\mathrm{C}_{28} \mathrm{H}_{23}{ }^{37} \mathrm{ClN}_{4} \mathrm{O}_{3} \mathrm{~S}\right), \quad 530.1159 \quad\left(\mathrm{C}_{28} \mathrm{H}_{23}{ }^{35} \mathrm{ClN}_{4} \mathrm{O}_{3} \mathrm{~S}\right), \quad 506.1007 \quad\left(\mathrm{C}_{26} \mathrm{H}_{21}{ }^{37} \mathrm{ClN}_{4} \mathrm{O}_{3} \mathrm{~S}\right), \quad 505.1089$ $\left(\mathrm{C}_{26} \mathrm{H}_{21}{ }^{35} \mathrm{ClN}_{4} \mathrm{O}_{3} \mathrm{~S}\right)$.

N-\{1-[[2-[5-(2-Fluorobenzylidene)-3-benzyl-4-oxo-1,3-thiazolidin-2-ylidene]hydrazino]carbonyl]-3-(methylthio)propyl $\}$ benzamide (26). Synthesis of compound $\mathbf{2 6}$ was carrried out by refluxing compound $11(0.456 \mathrm{~g}, 0.001 \mathrm{~mol})$ with 2-fluorobenzaldehyde $(98 \%, 0.126 \mathrm{~g}, 0.001$ mol) according to the procedure given above for compounds 12-27. Yield $61 \%$. M.p. $210-211^{\circ} \mathrm{C}$. HPLC $t_{R}(\mathrm{~min}): 12.450,15.018$. IR, v $\left(\mathbf{c m}^{-1}\right): 3267$ (br), 3045, 2913, 2838 (w), 1711, 1694, 1632, 1615 , 1579, 1569 (s), 1483, 1422 (m), 1383, 1356 (s), 1329, 1238 (w), 1213 (s), 1157 (w), 1111 (m), 961 (w). ${ }^{1} \mathbf{H}$ NMR : $\boldsymbol{\delta}$ (ppm) 2.04, $2.20,2.27-2.35$ (s, s, m, 5H), 2.43-2.61, 2.62-2.78 and 2.79-2.93 ( $\mathrm{m}, \mathrm{m}$ and $\mathrm{m}, 2 \mathrm{H}), 4.88-5.01$ and 5.47-5.61 (q and $\mathrm{m}, 1 \mathrm{H}$ ), 5.01-5.28 $(5 \mathrm{~s}, 2 \mathrm{H}), 7.14-7.36(\mathrm{~m}, 6 \mathrm{H}), 7.42-7.57(\mathrm{~m}, 7 \mathrm{H}), 7.85(\mathrm{~d}, 2 \mathrm{H}, J=7.7 \mathrm{~Hz}), 8.02$ and 8.11 (s and s, 1H), 7.74 and 9.31 ( $\mathrm{s}$ and s, $1 \mathrm{H}$ ). HR-MS (EI $\left.{ }^{+}\right), \mathbf{m} / \mathbf{z}: 562.1526\left(\mathrm{M}^{+}, \mathrm{C}_{29} \mathrm{H}_{27} \mathrm{~F} \mathrm{~N}_{4} \mathrm{O}_{3} \mathrm{~S}_{2}\right.$ requires 562.1527), $514.1464\left(\mathrm{C}_{28} \mathrm{H}_{23} \mathrm{FN}_{4} \mathrm{O}_{3} \mathrm{~S}\right), 488.1318\left(\mathrm{C}_{26} \mathrm{H}_{21} \mathrm{FN}_{4} \mathrm{O}_{3} \mathrm{~S}\right), 327.0848\left(\mathrm{C}_{17} \mathrm{H}_{14} \mathrm{FN}_{3} \mathrm{OS}\right)$, $312.0745\left(\mathrm{C}_{17} \mathrm{H}_{13} \mathrm{FN}_{2} \mathrm{OS}\right), 105.0336\left(\mathrm{C}_{7} \mathrm{H}_{5} \mathrm{O}\right)$.

N-\{1-[[2-[5-(4-Fluorobenzylidene)-3-benzyl-4-oxo-1,3-thiazolidin-2-ylidene]hydrazino]carbonyl]-3-(methylthio)propyl $\}$ benzamide (27). Synthesis of compound 27 was carrried out 
by refluxing compound $11(0.456 \mathrm{~g}, 0.001 \mathrm{~mol})$ with 4-fluorobenzaldehyde $(0.124 \mathrm{~g}, 0.001 \mathrm{~mol})$ according to the procedure given above for compounds 12-27. Yield 76\%. M.p. 230-231 ${ }^{\circ} \mathrm{C}$. HPLC $t_{R}(\min ): 14.150,15.284 . \mathbf{I R}, \boldsymbol{v}\left(\mathbf{c m}^{-1}\right): 3233(\mathrm{~N}-\mathrm{H}, \mathrm{br}), 3061,3036,2982,2921,2838$ (w), 1715, 1665 (s), 1638 (vs), 1603, 1567 (s), 1512, 1431 (m), 1387, 1339 (s), 1312 (w), 1238 (s), 1183, 1162, 828 (C-H, m). ${ }^{1} \mathbf{H}$ NMR : $\boldsymbol{\delta}$ (ppm) 1.84, 2.01, 2.08-2.16 (s, s, m, 5H), 2.182.30, 2.43-2.58 and 2.58-2.75 (m, $\mathrm{m}$ and $\mathrm{m}, 2 \mathrm{H}), 4.77-4.79$ and 5.26-5.42 (q and $\mathrm{m}, 1 \mathrm{H}), 4.80-$ $5.03(5 \mathrm{~s}, 2 \mathrm{H}), 6.94-7.03(\mathrm{~m}, 3 \mathrm{H}), 7.10-7.15(\mathrm{~m}, 3 \mathrm{H}), 7.23-7.37(\mathrm{~m}, 8 \mathrm{H}), 7.65(\mathrm{~d}, 2 \mathrm{H}, J=$ $7.3 \mathrm{~Hz}$ ), 7.54 and 9.21 ( $\mathrm{s}$ and $\mathrm{s}, 1 \mathrm{H})$. HR-MS $\left(\mathbf{E I}^{+}\right), \mathbf{m} / \mathbf{z}: 562.1500\left(\mathrm{M}^{+}, \mathrm{C}_{29} \mathrm{H}_{27} \mathrm{FN}_{4} \mathrm{O}_{3} \mathrm{~S}_{2}\right.$ requires 562.1527), $514.1661 \quad\left(\mathrm{C}_{28} \mathrm{H}_{23} \mathrm{FN}_{4} \mathrm{O}_{3} \mathrm{~S}\right), \quad 488.1331 \quad\left(\mathrm{C}_{26} \mathrm{H}_{21} \mathrm{FN}_{4} \mathrm{O}_{3} \mathrm{~S}\right), \quad 327.0848$ $\left(\mathrm{C}_{17} \mathrm{H}_{14} \mathrm{FN}_{3} \mathrm{OS}\right), 312.0742\left(\mathrm{C}_{17} \mathrm{H}_{13} \mathrm{FN}_{2} \mathrm{OS}\right), 105.0334\left(\mathrm{C}_{7} \mathrm{H}_{5} \mathrm{O}\right)$.

\section{Antibacterial assay}

Newly synthesized compounds were individually tested against laboratory strains of totally 64 microbial culture isolates of 56 bacteria and 7 fungi and 1 yeast species which described previously ${ }^{17}$ were tested by using disc-diffusion, micro-well dilution assay ${ }^{41,42}$ and MIC agar dilution assay ${ }^{43}$.Microorganisms were provided by the Department of Biology, Faculty of Art and Science, and at Atatürk University, Erzurum, Turkey. The identity of the microorganisms used in this study was confirmed by Microbial Identification System in Biotechnology Application and Research Center at Atatürk University.

\section{Antiviral assay}

Evaluation of the antiviral activity of the compounds against HIV-1 strain $\mathrm{III}_{\mathrm{B}}$ and HIV-2 strain (ROD) in MT-4 cells was performed using the MTT assay as previously described ${ }^{44}$. HIV1 (IIIB) ${ }^{45}$ or HIV-2 (ROD) ${ }^{46}$ stock $(50 \mu \mathrm{l})$ at 100-300 CCID $_{50}$ (cell culture infectious dose) or culture medium was added to either the infected or mock-infected wells of the microtiter tray. Mock-infected cells were used to evaluate the effect of test compound on uninfected cells in order to assess the cytotoxicity of the test compound. Exponentially growing MT-4 cells ${ }^{47}$ were centrifuged for 5 minutes at $1000 \mathrm{rpm}$ and the supernatant was discarded. The MT-4 cells were resuspended at $6 \times 10^{5} \mathrm{cells} / \mathrm{ml}$, and $50-\mu 1$ volumes were transferred to the microtiter tray wells. Five days after infection, the viability of mock-and HIV-infected cells was examined spectrophotometrically by the MTT assay. The antiviral assays, other than HIV-1, were based on inhibition of virus-induced cytopathicity in human embryonic lung (HEL) cells (HSV-1, HSV-2 , Vaccinia virus, Vesicular stomatitis virus), HeLa cells (Vesicular stomatitis virus, Coxsackie virus, Respiratory syncytial virus) or Vero cells (Parainfluenza-3 virus, Sindbis virus, Punta Toro virus, Reovirus-1, Coxsackie virus B4), following previously established procedures. $^{48-50}$

\section{Antimycobacterial assay}

The primary screen was conducted at $6.25 \mu \mathrm{g} \mathrm{mL}^{-1}$ against M. tuberculosis H37Rv in BACTEC 12B medium using a broth microdilution assay, the Microplate Alamar Blue Assay (MABA). 
Compounds exhibiting fluorescence were tested in the BACTEC 460 radiometric system. $^{51}$ Compounds effecting $<90 \%$ inhibition in the primary screen (MIC $>6.25 \mu \mathrm{g} \mathrm{mL}^{-1}$ ) were not further evaluated. Compounds demonstrating at least $90 \%$ inhibition in the primary screen were re-tested at lower concentrations against $\mathrm{M}$. tuberculosis $\mathrm{H} 37 \mathrm{Rv}$ to determine the actual minimum inhibitory concentration (MIC) in the MABA. The MIC was defined as the lowest concentration inhibiting $99 \%$ of the inoculum.

\section{Anticancer assay}

A total of 60 human tumor cell lines derived from nine cancer types (non-small cell lung, colon, breast, ovarian, leukemia, renal, prostate, CNS, melanoma) formed the basis of this test. The tumor cells were cultured in RPMI1640 medium supplemented with 5\% fetal calf serum and $2 \mathrm{mM}$ L-glutamine. The tumor cells are inoculated over a series of standard 96-well microtiter plates in $100 \mu$ l of medium. ${ }^{52-53}$ Density of the inoculum depended on the types of tumor cells and their growth characteristics. ${ }^{54}$ These cells are then preincubated on the microtiter plate for $24 \mathrm{~h}$ before adding the compounds. These were tested starting from DMSO solutions at five different concentrations $\left(10^{-4}, 10^{-5}, 10^{-6}, 10^{-7}, 10^{-8} \mathrm{M}\right)$. After an incubation of the chemical agent for $48 \mathrm{~h}$ with the tumor cell lines, sulforhodamine B (SRB) protein assay was used to estimate cell viability and growth. The cytotoxic effects are evaluated and the assay results and doseresponse parameters were calculated as described. ${ }^{55}$ Details of this test system and the information derived from the activity pattern over all cell lines have been published. ${ }^{54,56-57}$

\section{Acknowledgements}

The authors are grateful to Dr. Jürgen Gross from the Institute of Organic Chemistry, University of Heidelberg, for his generous help on obtaining HR-EI/FAB mass spectra of the synthesized compounds. We also thank Dr. Joseph A. Maddry from the Tuberculosis Antimicrobial Acquisition and Coordinating Facility (TAACF), National Institute of Allergy and Infections Diseases Southern Research Institute, GWL Hansen's Disease Centre, Colorado State University, Birmingham, AL, USA, for the in vitro evaluation antimycobacterial activity using M. tuberculosis H37Rv. We thank the members of Drug Research and Development, Division of Cancer Research, National Cancer Institute, Bethesda, Maryland, USA, for the anticancer activity screening of the compounds. Prof. Erik De Clercq thanks Prof. Christophe Pannecouque for his invaluable help in obtaining the anti-HIV data and Mrs. Leentje Persoons for her technical assistance in obtaining the other antiviral data. 


\section{References}

1. Clarck, C.; Jacobs, M.; Appelbaum, P. J. Clin. Microbiol. 1998, 36, 3759.

2. Davidson, P. T. ;Le, H. Q. Drugs 1992, 43, 651.

3. Snider, D. E.Jr.; Raviglione, M.; Kochi, A. Global Burden of Tuberculosis, in : Tuberculosis : Pathogenesis, Protection and Control, Bloom, B. Eds.; ASM : Washington, D.C., $1994 ;$ p 3.

4. Babaoglu, K.; Page, M. A.; Jones, V. C.; McNeil, M. R.; Dong, C.; Naismith, J. H.; Lee, R. E. Bioorg. Med. Chem. Lett. 2003, 13, 3227.

5. Turner, B.; Summers, M. F. J. Mol. Biol. 1999, 285, 1.

6. De Clercq, E. Nature Reviews Drug Discovery 2002, 1, 13.

7. De Clercq, E. AIDS Res. Hum. Retrovirus 1992, 8, 119.

8. Jonckheere, H.; Anné, J.; De Clercq, E. Med. Res. Rev. 2000, 20, 129.

9. Barreca, M. L.; Balzarini, J.; Chimirri A.; De Clercq, E.; De Luca, L.; Höltje, H. D.; Höltje, M.; Monforte, A. M.; Monforte, P.; Pannecouque, C.; Rao, A.; Zappalà, M. J. Med. Chem. 2002, 45, 5410 .

10. De Clercq, E. Nature Reviews Drug Discovery 2007, 6, 1001.

11. Ahgren, C.; Backro, K.; Bell, F. W.; Cantrell, A. S.; Clemens, M.; Colacino, J. M.; Deeter, J. B.; Engelhardt, J. A.; Hogberg, M.; Jaskunas, S. R.; Johansson, N. G.; Jordan, C. L.; Kasher, J. S.; Kinnick, M. D.; Lind, P.; Lopez, C.; Morin, J. M.; Muesing, M. A.; Noreen, R.; Oberg, B.; Paget, C. J.; Palkowitz, J. A.; Parrish, C. A.; Pranc, P.; Rippy, M. K.; Rydergard, C.; Sahlberg, C.; Swanson, S.; Ternansky, R. J.; Unge, T.; Vasileff, R. T.; Vrang, L.; West, S. J.; Zhang, H.; Zhou, X. X. Antimicrob. Agents Chemother. 1995, 39, 1329.

12. Barreca, M. L.; Chimirri, A.; De Luca, L.; Monforte, A. M.; Monforte, P.; Rao, A.; Zappalà, M.; Balzarini, J.; De Clercq, E.; Pannecouque, C.; Witvrouw, M. Bioorg. Med. Chem. Lett. 2001, 11, 1793.

13. Andres, C. J.; Bronson, J. J.; D’Andrea, S. V.; Deshpande, M. S.; Falk, P. J.; Grant-Young, K. A.; Harte, W. E.; Ho, H.; Misco, P. F.; Robertson, J. G.; Stock, D.; Sun, Y.; Walsh, A. W. Bioorg. Med. Chem. Lett. 2000, 10, 715.

14. Bonde, C. G.; Gaikwad, N. J. Bioorg. Med. Chem. 2004, 12, 2151.

15. Karal1, N. ; Gürsoy, A.; Kandemirli, F.; Shvets N.; Kaynak F. B. ; Özbey S.; Kovalishyn V.; Dimoglo A. Bioorg. Med. Chem. 2007, 15, 5888.

16. Kü̧̈ükgüzel, Ş. G.; Oruç, E. E.; Rollas, S.; Şahin, F.; Özbek, A. Eur. J. Med. Chem. 2002, 37, 197.

17. Küçükgüzel, Ş. G.; Kocatepe A.; De Clercq E.; Şahin, F.; Güllüce M. Eur. J. Med. Chem. 2006, 41, 353.

18. Rao, A.; Carbone, A.; Chimirri, A.; De Clercq, E.; Monforte, A. M.; Monforte, P.; Pannecouque, C. ; Zappalà, M. Farmaco 2003, 58, 115.

19. Barreca, M. L.; Chimirri, A.; De Clercq, E.; De Luca, L.; Monforte, A. M.; Monforte, P.; Rao, A.; Zappalà, M. Farmaco 2003, 58, 259. 
20. Rao, A.; Balzarini, J. ; Carbone, A.; Chimirri, A.; De Clercq, E.; Monforte, A. M. ; Monforte, P. ; Pannecouque, C. ; Zappalà, M. Antiviral Res. 2004, 63, 79.

21. Rawal, R. ; Yenamandra, S. P. ; Katti, S. B. ; De Clercq, E. Bioorg. Med. Chem. 2005, 13, 6771.

22. Kaushik-Basu, N. ; Bopda-Waffo, A. ; Talele, T.T. ; Basu, A. ; Chen, Y. ; Küçükgüzel, Ş. G. Frontiers in Bioscience. 2008, 13, 3857.

23. Rawal, R.; Tripathi, R.; Katti, S. B.; Pannecouque, C.; De Clercq, E. Bioorg. Med. Chem. 2007, 15, 1725.

24. Ramla, M. M.; Omar, M. A.; Tokuda, H.; El-Diwani, H. I. Bioorg. Med. Chem. 2007, 15, 6489.

25. Ottanà, R.; Carotti, S.; Maccari, R.; Landini, I.; Chiricosta, G.; Caciagli, B.; Vigorita, M. G.; Mini, E. Bioorg. Med. Chem. Lett. 2005, 15, 3930.

26. Gududuru, V.; Hurh, E.; Dalton, J. T.; Miller, D. D. J. Med. Chem. 2005, 48, 2584.

27. Gududuru, V.; Hurh, E.; Dalton, J. T.; Miller, D. D. Bioorg. Med. Chem. Lett. 2004, 14 , 5289.

28. Mills, T.; Roberson, J. C. In Instrumental Data for Drug Analysis; Fischer, B. A. J., Ed., $2^{\text {nd }}$ Ed. Elsevier: New York, 1987.

29. Marković, R.; Pergal, M. M.; Baranac, M.; Stanisavljev, D.; Stojanović, M. ARKIVOC 2006, (ii), 83 .

30. Bondock, S.; Khalifa, W.; Fadda, A. A. Eur. J. Med. Chem. 2007, 42, 948.

31. Ashok, M.; Holla, B. S.; Kumari, N. S. Eur. J. Med. Chem. 2007, 42, 380.

32. Şahin, G.; Palaska, E.; Kelicen, P.; Demirdamar, R.; Altınok, G. Arzneim.-Forsch.। Drug Res. 2001, 51, 478.

33. Cesur, Z.; Ergenç, N.; İlhan, E. Acta Pharmaceutica Turcica 1989, 31, 103.

34. Archana; Srivastava, V. K. ; Kumar, A. Eur. J. Med. Chem. 2002, 37, 873.

35. Çapan, G. ; Ulusoy, N.; Ergenç, N.; Kiraz, M. Monatschefte für Chemie 1999, 130, 1399.

36. Ulusoy, N.; Çapan, G.; Ergenç, N.; Ekinci, A. C.; Vidin A. Acta Pharmaceutica Turcica 1998, 40, 5.

37. Ergenç, N.; Çapan, G. Il Farmaco 1994, 49, 133.

38. Tozkoparan, B.; Akgün, H.; Ertan, M.; Rübsamen, K. Arch. Pharm. (Weinheim) 1995, 328, 169.

39. Kameda, Y.; Toyoura, E.; Kimura, Y.; Kanaya, Y.; Yoshimura, K. Enzymic resolution of methionine and cystine. Yakugaku Zasshi 1958, 78, 748-753; Ref. Chem. Abstr. 1958, 52, 18237.

40. Nakagawa, M.; Suzuki, T.; Kawashima, T.; Hino, T. Oxidation of methionine to benzoyl methionine sulfoxide with 3-hydroperoxyindolenines. Chem. Pharm. Bull.1972, 20, 24132417.

41. Şahin, F.; Karaman, İ.; Güllüce, M.; Öğütçü, H.; Şengül, M.; Adıgüzel, A.; Öztürk, S.; Kotan, R. J. Ethnopharmacology 2002, 87, 61. 
42. Güllüce, M.; Adıgüzel, A; Öğütçü, H.; Şengül, M.; Şahin, F. Phytotherapy Res. 2004, 18, 208.

43. Güllüce, M.; Sökmen, M.; Şahin, F.; Sökmen, A.; Adıüzel, A.; Özer, H. J. Sc. Food Agriculture 2004, 84, 735.

44. Pauwels, R. ; Balzarini, J.; Baba, M.; Snoeck, R.; Schols, D.; Herdewijn, P.; Desmyter, J.; De Clercq, E. J. Virol. Methods. 1988, 20, 309.

45. Popovic, M.; Sarngadharan, M. G.; Read, E.; Gallo, R. C. Science 1984, 224, 497.

46. Barré-Sinoussi, F.; Chermann, J. C.; Nugeyre, M. T.; Chamaret, S.; Grest, J.; Dauget, C.; Axler-Blin, C.; Vézinet-Brun, F.; Rouzioux, C.; Rozenbaum, W.; Montagnier, L. Science 1983, 220, 868 .

47. Miyoshi, I.; Taguchi, H.; Kobonishi, I.; Yoshimoto, S.; Ohtsuki, Y.; Shiraishi, Y.; Akagi, T.; Gann. Monogr. 1982, 28, 219.

48. De Clercq, E.; Descamps, J.; Verhelst, G.; Walker, R. T.; Jones, A. S.; Torrence, P. F.; Shugar, D. J. Infect. Dis. 1980, 141, 563.

49. De Clercq, E.; Holý, A.; Rosenberg I.; Sakuma, T.; Balzarini, J.; Maudgal, P.; C. Nature 1986, 323, 464.

50. De Clercq, E. Antimicrob.Agents Chemother. 1985, $28,84$.

51. Collins, L.; Franzblau, S. G. Antimicrob.Agents.Chemother. 1997, 41, 1004.

52. Grever, M. R.; Schepartz, S. A.; Chabner, B. A. Semin. Oncol. 1992, 19, 622.

53. Boyd, M. R.;Paull, K. D. Drug Dev. Res. 1995, 34, 91.

54. Weinstein, J. N.; Myers, T. G.; O’Connor, P. M.; Friend, S. H.; Fornance, A. J.; Kohn, K. W.; Fojo, T.; Bates, K.; Van Osdol, W. W.; Monks, A. P.; Scudiero, D. A.; Sausville, E. A.; Zaharevitz, D. W.; Bunow, B.; Viswanadhan, V. N.; Johnson, G. S.; Wittes, R. E.; Paull, K. D. Science. 1997, 275, 343.

55. Boyd, M. R. Princ. Pract. Oncol. 1989, $3,1$.

56. Boyd, M. R. Am. Assoc.Cancer Res. 1989, 30, 652.

57. Monks, A. P.; Scudiero, D. A.; Shekan, P.; Shoemaker, K. D.; Paull, D.; Vistica, C.; Hose, C.; Langley, J.; Cronise, P.; Vaigro -Wolff, A.; Gray-Goodrich, M.; Cambell, H.; Mayo, M.; Boyd, M. J. Natl. Cancer Inst. 1991, 83, 757. 